Ecología

\title{
Nymphalidae frugívoras (Lepidoptera: Papilionoidea) asociadas a un ecomosaico agropecuario y de bosque tropical lluvioso en un paisaje del sureste de México
}

\author{
Frugivorous Nymphalidae (Lepidoptera: Papilionoidea) associated to an ecomosaic of agriculture \\ and tropical rainforest in a landscape in Southeastern Mexico \\ Noel Antonio González-Valdivia ${ }^{\mathrm{a}}$, Carmen Pozo ${ }^{\mathrm{b}}$, Susana Ochoa-Gaona ${ }^{\mathrm{c}, *}$, \\ Bruce Gordon Ferguson ${ }^{\mathrm{d}}$, Eduardo Cambranis ${ }^{\mathrm{a}}$, Orlando Lara ${ }^{\mathrm{a}}$, Isidro Pérez-Hernández ${ }^{\mathrm{e}}$, \\ Alejandro Ponce-Mendoza ${ }^{\mathrm{f}}$ y Christian Kampichler ${ }^{\mathrm{g}}$ \\ a Departamento de Ingenierías, Instituto Tecnológico de Chiná, Tecnológico Nacional de México. Calle 11 s/n entre 22 y 28, 24520, Chiná, Campeche, México \\ ${ }^{\mathrm{b}}$ Departamento de Conservación de la Biodiversidad, El Colegio de la Frontera Sur, Av. del Centenario km. 5.5, Pacto obrero campesino, 77014, \\ Chetumal, Quintana Roo, México \\ ${ }^{\mathrm{c}}$ Departamento de Ciencias de la Sustentabilidad, El Colegio de la Frontera Sur, Av. Rancho, Polígono 2-A, Parque Industrial de Lerma, 24500, Campeche, \\ Campeche, México \\ ${ }^{\mathrm{d}}$ Departamento de Agricultura, Sociedad y Ambiente, El Colegio de la Frontera Sur, Carretera Panamericana y Periférico Sur s/n, María Auxiliadora, 29290, \\ San Cristóbal de las Casas, Chiapas, México \\ ${ }^{\text {e } U n i v e r s i d a d ~ P o l i t e ́ c n i c a ~ d e l ~ G o l f o ~ d e ~ M e ́ x i c o, ~ C a r r e t e r a ~ F e d e r a l ~ M a l p a s o-E l ~ B e l l o t e ~ k m . ~} 17$ Ranchería Monte Adentro, 86600, Paraíso, Tabasco, México \\ ${ }^{\mathrm{f}}$ Centro Nacional de Investigación Disciplinaria en Conservación y Mejoramiento de Ecosistemas Forestales, INIFAP. Av. Progreso \#5, Colonia Barrio de Santa \\ Catarina, 04010, Ciudad de México, México \\ ${ }^{\mathrm{g}}$ Sovon Dutch Centre for Field Ornithology, Natuurplaza (Mercator 3), Toernooiveld 1, 6525 ED, Nijmegen, Países Bajos
}

Recibido el 19 de mayo de 2015; aceptado el 22 de enero de 2016

Disponible en Internet el 24 de mayo de 2016

\section{Resumen}

Se evaluó si la coloración de las Nymphalidae frugívoras es un indicador de las unidades del paisaje. Se consideraron 4 condiciones de matriz agropecuaria (vegetación secundaria madura, vegetación secundaria joven, pastizales y cercos vivos) comparadas con 4 unidades de bosque (referente ecológico), en Tenosique, Tabasco, México. En cada unidad de paisaje se establecieron 10 trampas Van Someren-Rydon durante 5 días, aplicando 4 repeticiones. Se identificaron 63 especies, 58 en la matriz agropecuaria y 42 en el referente ecológico. Treinta y siete especies son compartidas, 21 asociadas a áreas agropecuarias y 5 solo del referente ecológico. La estructura de la vegetación afectó la abundancia y composición, pero no la riqueza de mariposas. Las comunidades de la matriz agropecuaria difirieron $>75 \%$ respecto al referente ecológico. Hubo correlación entre el color y el hábitat preferencial. Los patrones tigre y transparente (Danainae) se encontraron en el dosel cerrado. Con excepción de Adelpha (Limenitidinae) y las de color corteza (Biblidinae), las demás utilizan ampliamente el paisaje, destacando el tipo Hamadryas asociado a ambientes abiertos. El mosaico del paisaje estudiado de remanentes de bosque asociados a una matriz agropecuaria arbolada favorece la conservación del gremio de lepidópteros y mantiene una alta diversidad de las Nymphalidae frugívoras.

Derechos Reservados (C) 2016 Universidad Nacional Autónoma de México, Instituto de Biología. Este es un artículo de acceso abierto distribuido bajo los términos de la Licencia Creative Commons CC BY-NC-ND 4.0.

Palabras clave: Mariposas diurnas; Biodiversidad; Indicador ecológico; Preferencias de hábitat; Neotrópico

\section{Abstract}

We evaluated the use of coloring of frugivorous Nymphalidae as an indicator of landscape units. Four environmental matrices were taken into consideration (old secondary vegetation, young secondary vegetation, pasture and live fences) and compared to four forest units (ecological reference), in Tenosique, Tabasco, México. In each landscape unit we set up 10 Van Someren-Rydon traps for 5 days, replicating the sampling

\footnotetext{
* Autor para correspondencia.

Correo electrónico: sochoa@ecosur.mx (S. Ochoa-Gaona).

La revisión por pares es responsabilidad de la Universidad Nacional Autónoma de México.
} 
4 times. In total 63 species were identified, with 58 in the agricultural matrix and 42 in forested units. Of the total, 37 species were shared, 21 associated to agricultural areas and 5 to the forest units. The structure of the vegetation affected the species abundance and composition, but not the butterfly richness. The communities of butterflies in pasture lands and in fallows differ $>75 \%$ of those of the forest units. There was correlation between color and preferential habitat. Tiger and transparent patterns (Danainae) prefer the closed canopy. Excepting Adelpha (Limenitidinae,) and those with bark colour (Biblidinae), all others are widely distributed in the landscape, particularly the Hamadryas type were associated with open environments. The landscape resulting from management, where forest remnants are mixed with agricultural plots and secondary vegetation in various stages of succession, seems to favor the conservation of Lepidoptera and to maintain a high diversity of frugivorous Nymphalidae.

All Rights Reserved (C 2016 Universidad Nacional Autónoma de México, Instituto de Biología. This is an open access item distributed under the Creative Commons CC License BY-NC-ND 4.0.

Keywords: Butterflies; Biodiversity; Ecological indicator; Habitat preferences, Neotropics

\section{Introducción}

Entre los estudios sobre la dinámica de poblaciones de las especies destacan los enfoques derivados de la teoría de biogeografía de islas (MacArthur y Wilson, 1967), de metapoblaciones (Hanski, 1998; Hanski y Gilpin, 1996) y la noción de equilibrio dinámico entre procesos demográficos (Simberloff, 1974). En la mayoría de estas investigaciones, el ser humano es separado del análisis de procesos naturales y a menudo considerado un agente antagónico a los ecosistemas. Desde inicios de la década de 1990, los estudios de diversidad en paisajes han evidenciado la capacidad de la flora y fauna de adaptarse a la influencia humana y aún de recuperarse después de periodos prolongados de presión antropogénica (Apaza, Osorio y Pastrana, 2006; Harvey et al., 2008; Harvey y Haber, 1999).

Algunos ecosistemas bajo manejo humano mantienen alta diversidad de especies (Hartshorn, 1995), resultado de intervenciones antrópicas de intensidad y duración moderadas, que promueven paisajes heterogéneos en su estructura. En otras palabras, un paisaje que conserva vegetación original alternada con coberturas modificadas por la actividad humana puede conservar y mantener la biodiversidad nativa. En América tropical, la agricultura migratoria o «milpa tradicional» genera paisajes con esas características (Gómez-Pompa y Kaus, 1999). Este manejo del sistema, basado en el conocimiento local, puede considerarse como un proceso de interacción entre sistemas socioeconómicos y ecológicos sustentables (Coomes, Grimard y Burt, 2000; Higgs, 2003; Holling, 2001). Sin embargo, a partir de las décadas de 1940 y 1950, se ha incrementado la intensidad en el uso del suelo acortando los periodos del barbecho e impidiendo el retorno al estado de bosque maduro. Actualmente, la explotación de la tierra con fines comerciales ha modificado estos sistemas tradicionales, disminuyendo la capacidad de conservación ecológica del sistema (DeFries, Rudel, Uriarte y Hansen, 2010; Ford y Nigh, 2010; Fox et al., 2000).

En el sureste mexicano es posible que los paisajes puedan aún ser compatibles con la conservación biológica y con los propósitos económicos y productivos de los seres humanos. Para evaluar esto, se han empleado a las mariposas Nymphalidae frugívoras como herramienta ecológica sobre la idoneidad de los hábitats. Este grupo es útil por su respuesta diferenciada y dependiente de cada especie a los efectos de los distintos niveles de alteración ambiental (Atauri y de Lucio, 2001; Kitching et al., 2000; Waltz, Covington y Wallace, 2001; Weibull, Bengtsson y Nohlgren,
2000). Por otra parte, además de ser especies indicadoras, las mariposas tropicales tienen funciones importantes en los ecosistemas tropicales (Bonebrake, Ponisio, Boggs y Ehrlich, 2010).

Las especies frugívoras de la familia Nymphalidae (Lepidoptera: Papilionoidea) son un componente importante de la fauna tropical (Heppner, 2002). Aunque son conocidas como frugívoras, realmente se alimentan de fluidos fermentados de frutas, de estiércol o de lodos; para fines del artículo, nos referiremos a estas como frugívoras (Santos, Iserhard, Teixeira y Romanowski, 2011). A diferencia de lo propuesto por Freitas et al. (2014), consideramos como frugívoras también a algunas especies de otras subfamilias como Limenitidinae y Biblidinae, de acuerdo con las abundancias registradas en este trabajo y así consideradas por otros autores (Krenn, Zulka y Gatschnegg, 2001; Pinheiro y Ortiz, 1992; Willmott, 2003). La composición y abundancia de las Nymphalidae se modifican por la alteración del hábitat (Beck, Kitching y Linsenmair, 2006; Miller, 1994), por lo que pueden considerarse indicadoras de afectación del ambiente (Sparrow, Sisk, Ehrlich y Murphy, 1994; Ricketts, Daily, Ehrlich y Fay, 2001; Tobar, Ibrahim y Casasola, 2007). Asimismo, pueden indicar el estado de toda la comunidad de mariposas en una región (Horner-Devine, Daily, Ehrlich y Boggs, 2003). Además de ser un grupo bien conocido taxonómicamente, sus patrones de coloración críptica pueden estar relacionados con algunos hábitats en particular, lo que las hace útiles como indicadoras o para predecir los efectos causados por el disturbio (De la Maza y de la Maza, 1993; De la Maza y Soberón, 1998). El objetivo de este trabajo fue identificar si el paisaje que se produce por el manejo de los recursos en una localidad tropical mantiene una comunidad diversa de mariposas Nymphalidae frugívoras y si algunas de estas son indicadoras de las unidades de paisaje (UP) de acuerdo con su coloración.

\section{Materiales y métodos}

La comunidad de Niños Héroes de Chapultepec, Tenosique, Tabasco, ubicada en las coordenadas $17^{\circ} 15^{\prime} 00^{\prime \prime}-17^{\circ} 40^{\prime} 48^{\prime \prime} \mathrm{N}$ y 9059'09”-91³8'16" O (Ochoa-Gaona, Pérez-Hernández y de Jong, 2008) mantiene el sistema de tenencia y manejo ejidal de la tierra sobre un área de 2,066 ha. La precipitación promedio anual es de $2,750 \mathrm{~mm}$ y la temperatura media anual de $26{ }^{\circ} \mathrm{C}$ (Isaac-Márquez, de Jong, Eastmond, Ochoa-Gaona y Hernández, 2005). El relieve es montañoso con alturas máximas 
Tabla 1

Características ambientales y estructurales de las unidades del paisaje en la Sierra de Tenosique, Tabasco.

\begin{tabular}{|c|c|c|c|c|c|c|c|}
\hline \multirow[t]{2}{*}{ UP } & \multicolumn{3}{|c|}{ Variables ambientales } & \multicolumn{4}{|c|}{ Variables estructurales } \\
\hline & Alt & $\mathrm{T}$ & Ilu & $\mathrm{CD}$ & $\mathrm{AD}$ & $\mathrm{Da}$ & $\mathrm{AB}$ \\
\hline \multicolumn{8}{|l|}{$M A$} \\
\hline $\mathrm{AM}$ & $393 \pm 87$ & $27 \pm 1$ & $3,396 \pm 2,930$ & $80 \pm 8$ & $19 \pm 5$ & $1,125 \pm 253$ & $19 \pm 5$ \\
\hline AJ & $316 \pm 59$ & $28 \pm 1$ & $4,581 \pm 3381$ & $65 \pm 7$ & $9 \pm 3$ & $1,028 \pm 336$ & $13 \pm 5$ \\
\hline $\mathrm{AL}$ & $264 \pm 25$ & $30 \pm 2$ & $11,544 \pm 2,944$ & $31 \pm 8$ & $7 \pm 1$ & $357 \pm 143$ & $15 \pm 6$ \\
\hline PAD & $279 \pm 28$ & $32 \pm 2$ & $15,018 \pm 596$ & $11 \pm 5$ & $7 \pm 2$ & $63 \pm 35$ & $5 \pm 2$ \\
\hline \multicolumn{8}{|l|}{$R E$} \\
\hline REAM & $544 \pm 52$ & $25 \pm 1$ & $1,919 \pm 1,662$ & $84 \pm 1$ & $28 \pm 3$ & $1,145 \pm 81$ & $42 \pm 18$ \\
\hline REAJ & $526 \pm 85$ & $25 \pm 1$ & $2,519 \pm 1,326$ & $84 \pm 3$ & $29 \pm 1$ & $1,227 \pm 119$ & $37 \pm 14$ \\
\hline REPAD & $500 \pm 71$ & $25 \pm 1$ & $2,630 \pm 1,222$ & $83 \pm 6$ & $29 \pm 2$ & $1,088 \pm 33$ & $33 \pm 4$ \\
\hline REL & $505 \pm 97$ & $26 \pm 1$ & $2,564 \pm 235$ & $74 \pm 6$ & $29 \pm 4$ & $835 \pm 172$ & $25 \pm 14$ \\
\hline
\end{tabular}

AB: área basal ( $\left.\mathrm{m}^{2} / \mathrm{ha}\right) ; \mathrm{AD}$ : altura media del dosel (m); AJ: vegetación secundaria joven; Alt: altitud sobre el nivel del mar (m); AL: árboles en línea; AM: vegetación secundaria madura; Ilu: energía solar (MJ/m²/año); CD: cobertura del dosel (\%); Da: densidad de árboles (ind/ha); MA: matriz agropecuaria; PAD: potreros con árboles dispersos; RE: referente ecológico; REAJ: bosque referente para vegetación secundaria joven; REAM: bosque referente para vegetación secundaria maduras; REL: bosque alterado linealmente por rutas de acceso o senderos; REPAD: bosque referente para potreros con árboles dispersos; T: temperatura $\left({ }^{\circ} \mathrm{C}\right)$; UP: unidades del paisaje.

Adaptada de González-Valdivia et al. (2012).

de $700 \mathrm{~m}$ snm. Originalmente cubierta por vegetación de bosque tropical lluvioso (selva alta y mediana perennifolia). Los remanentes de bosque tropical se encuentran inmersos en un mosaico de vegetación secundaria de diferente edad, derivada de la actividad agrícola y pastizales inducidos para la ganadería localizados en las partes planas u onduladas (Isaac-Márquez et al., 2005). Estos remanentes de bosque tropical perennifolio -en su mejor estado- alcanzan los $30 \mathrm{~m}$ de altura y están conformados por 3 estratos arbóreos y un estrato herbáceo, compuestos por aráceas, marantáceas y helechos, además se presentan lianas y varios tipos de orquídeas (Sedespa, 1997). En el dosel predominan Aspidosperma cruentum, A. megalocarpon, Brosimum spp., Calophyllum brasiliense, Dialium guianense, Ficus spp., Lysiloma spp., Manilkara spp., Poulsenia armata, Pouteria spp. y Schizolobium parahyba. El sotobosque está dominado por Chamaedorea spp., Piper spp. y Psychotria spp. (González-Valdivia et al., 2012).

El patrón de paisaje en el ejido presenta 2 ecomosaicos (sensu Rossignol, Rossignol, Oldeman y Benzine-Tizroutine, 1998), uno formado por remanentes de bosque tropical lluvioso conservado, que sirve como referente ecológico (RE, sensu Higgs, 2003) y otro caracterizado por un patrón de fragmentos secuenciales de vegetación secundaria y potreros, al que denominamos matriz agropecuaria (MA). La reserva ejidal de bosque está protegida por la normatividad ejidal y forma parte del núcleo del Área Natural Protegida Cañón del Usumacinta en Tabasco, México, y se conecta al sur con El Petén, Guatemala y las estribaciones surorientales de la selva Lacandona, México (Semarnat, 2008).

\section{Diseño experimental}

Cada uno de los ecomosaicos se dividió en 4 UP siguiendo a Zonneveld (1989). Para MA se diferenció: 1) vegetación secundaria madura (AM) con más de 15 años de edad, la cual se ubicó en las partes más altas de las montañas; 2) vegetación secundaria joven $(\mathrm{AJ})$ con 8 a10 años de edad ubicada en las laderas y hacia la base de las montañas; 3) potreros con árboles dispersos (PAD) que se encuentran al pie de las montañas y en los valles, y 4) árboles en línea (AL), que forman cercos vivos alrededor de los potreros. En el ecomosaico RE se seleccionaron fragmentos de bosque ubicados de tal forma que fuera el referente para las UP de la MA. En la cima se ubicó la UP de bosque referente para vegetación secundaria madura (REAM), en las laderas el bosque referente de vegetación secundaria joven (REAJ), mientras al pie de monte y planicies se ubicaron las UP del bosque referente de potreros con árboles dispersos (REPAD) y de bosque alterado linealmente por rutas de acceso o senderos (REL). Cada UP se muestreó con 4 repeticiones.

En cada una de las UP se trazaron 12 parcelas rectangulares de $500 \mathrm{~m}^{2}$, donde se midieron variables de la vegetación en cuanto a su estructura y factores físico-ambientales. Las 12 variables evaluadas fueron: pendiente $(\%)$, temperatura ambiental $\left({ }^{\circ} \mathrm{C}\right)$, humedad relativa (\%), iluminación total, altitud, cobertura de dosel $(\%)$, cobertura del suelo (\%), número de estratos de vegetación, altura promedio del dosel (m), densidad arbustiva (ind/ha), densidad arbórea (ind/ha) y área basal $\left(\mathrm{m}^{2} / \mathrm{ha}\right)$. Se aplicaron correlaciones de Pearson, asumiéndose como colineales aquellas variables correlacionadas significativamente (Hernández, Barreto, Costa, Creão-Duarte y Favila, 2014). De este modo, las variables ambientales seleccionadas fueron: temperatura $\left({ }^{\circ} \mathrm{C}\right.$; medida con HOBO Data Logger y procesados utilizando Boxcar 3.0), altitud ( $\mathrm{m} \mathrm{snm}$; medida con altímetro con barómetro Casio Forester FTS-100), iluminación, registrada como la energía solar total captada a $1.3 \mathrm{~m}$ de la superficie del suelo $\left(\mathrm{MJ} / \mathrm{m}^{2} /\right.$ año; medido con fotos hemisféricas procesadas con Hemiview 2.1, Delta-T Devices, 1999), y variables estructurales: cobertura del dosel (\%; medida con densitómetro GRS) y altura media del dosel (m; tabla 1$)$.

El estudio se realizó de julio a septiembre de 2008, acumulando 40 días de muestreo, que de acuerdo con New (1998), son suficientes para capturar la mayor diversidad de mariposas. Además, en este periodo ocurre el mayor pico de fructificación de los árboles (Ochoa-Gaona et al., 2008), que al coincidir con una 
alta precipitación favorece la fermentación de frutos. Por ello, eran de esperar los incrementos en la densidad de Nymphalidae frugívoras (Pozo et al., 2008) y la presencia de la mayoría de las especies del gremio. Las UP del mismo tipo se establecieron con una distancia mínima de $1,000 \mathrm{~m}$ entre sí y, entre distintas UP, la separación mínima fue de $250 \mathrm{~m}$. En cada UP y sus repeticiones se establecieron transectos colocando trampas van Someren-Rydon cada $50 \mathrm{~m}$ a lo largo de $500 \mathrm{~m}$ de longitud (Pozo, Llorente-Bousquets, Luis-Martínez, Vargas-Fernández y Salas, 2005), las trampas se cebaron diariamente con fermento de piña, plátano y cerveza. En total se establecieron 10 trampas en un transecto o línea de trampas, permaneciendo 5 días consecutivos por $12 \mathrm{~h}$ diurnas/trampa. El muestreo se repitió 4 veces para cada unidad del paisaje ( $600 \mathrm{~h} /$ trampa por repetición). En total, el esfuerzo de muestreo fue de 2,400 h/trampa por UP, que corresponde a 20 días de $12 \mathrm{~h} /$ día/trampa por ecomosaico, totalizando $19,200 \mathrm{~h} /$ trampa para el paisaje en estudio y sus 8 unidades distintivas.

Todos los individuos capturados fueron identificados hasta especie o subespecie por especialistas del Museo de Zoología de El Colegio de la Frontera Sur (ECOSUR) y depositados en la Colección de Lepidoptera (ECOCH L, Registro ante INE: QNR.IN.018.0497) de ECOSUR, Chetumal, Quintana Roo, México. La nomenclatura de las especies se hizo con base en Lamas (2004) y la clasificación de acuerdo con Wahlberg et al. (2009).

El esfuerzo de muestreo fue evaluado mediante curvas de rarefacción Coleman, utilizando EstimateS 9.1.0 (Colwell, 2013), las cuales permiten la obtención de la asíntota mediante la ecuación de Clench (1979), calculando así el valor esperado de especies además de posibilitar la comparación de la riqueza entre cada UP y el paisaje, al tener este último un mayor número de muestras. La diversidad alfa para cada UP fue medida a través de índices de diversidad de Shannon-Wiener, dominancia de Simpson y equitatividad de Pielou (Spellerberg, 2005). Siguiendo la propuesta de Ramos (2004), se establecieron diferencias entre la abundancia para cada UP, mediante el análisis de varianza y la prueba de separación de medias de Tukey, previa determinación de normalidad y homocedasticidad mediante pruebas de Kolmogorov-Smirnov y de Levene, respectivamente. Se correlacionaron la riqueza (número de especies en una comunidad [McGinley, 2014; Stiling, 1999]) y la abundancia de especies de mariposas (número de individuos de cada especie en una comunidad [McGill et al., 2007]) con las variables estructurales y físico-ambientales de las UP una vez que se eliminaron las variables colineales de manera previa (Hernández et al., 2014).

La abundancia se clasificó en una escala de 5 categorías (modificado de New, 1998): dominantes (21 o más individuos y presentes en todas las localidades muestreadas), abundantes (11-20), comunes (6-10), ocasionales (2-5) y raras, cuando solo un individuo es capturado durante todo el muestreo en MA o en RE.

\section{Diversidad beta y relaciones ecológicas}

Previamente a los análisis multivariados para determinar la existencia de relaciones ecológicas y estudiar la diversidad beta, se examinaron los datos de abundancia de las especies y subfamilias mediante la prueba de Box y Cox (1964) para evaluar la posible transformación de los datos crudos para mejorar su ajuste a modelos matemáticos lineales. Con base en esta prueba, a la abundancia por especie se aplicó la transformación doble raíz, según indica la Ley de Taylor (Herrando-Pérez, 2002) y la transformación $\log (\mathrm{x}+1)$ en el caso de la abundancia para las subfamilias.

Se comparó la composición (es la identidad y variedad de los elementos de una comunidad, que incluye las especies que están presentes y su número [Conabio, 2009]) entre unidades a través de análisis de apareamiento de medias aritméticas no ponderadas (UPGMA) con el índice asimétrico de Bray-Curtis como medida de distancia que considera el valor cuantitativo del dato (Zuur, Ieno y Smith, 2007).

Mediante análisis de correspondencia canónica (ACC), se establecieron las relaciones de las comunidades, subfamilias y categorías de color de Nymphalidae respecto a las variables estructurales y ambientales que no estuvieron correlacionadas, como sugieren Araujo (2000) y Legendre (2008). Este análisis involucró validar las propuestas de De la Maza y de la Maza (1993) y de De la Maza y Soberón (1998) determinando si hay correspondencia entre los patrones de coloración de imagos y el grado de alteración, representada por las UP. Es importante mencionar que para las relaciones con las subfamilias de Nymphalidae, en este análisis se consideró la subfamilia Morphinae separada de la Satyrinae, con el propósito de conocer por separado la correlación de estos 2 grupos de especies.

Para corroborar el planteamiento de De la Maza y Soberón (1998), que señalan que ciertos grupos de mariposas pueden estar consistentemente asociadas con ciertos tipos de hábitats, se diferenciaron las siguientes categorías o patrones de coloración: adelpha (coloración típica del género Adelpha: Limenitidinae), bandas longitudinales, banda oblicua, corteza (similar a la corteza de los árboles, típico en Hamadryas: Biblidinae), follaje, naranja del dosel, neptis (coloración típica del género Neptis: Limenitidinae), negro/rojo, oscuro críptico, reflectante del dosel, reflectante del sotobosque, tigre y transparente (De la Maza y de la Maza, 1993; De la Maza y Soberón, 1998; González-Valdivia, Ochoa-Gaona, Pozo y Dénommée-Patriganni, 2010).

Los ambientes que representan cada una de las unidades de la MA y del RE en el paisaje fueron diferenciados mediante análisis de similitud (Anosim) y se identificaron las especies y subfamilias características de los mismos mediante el análisis de similitud porcentual (Simper). Las pruebas multivariadas fueron realizadas con los programas PAST 2.0 (Hammer, Harper y Ryan, 2001) y PRIMER 6.0 (Clarke y Gorley, 2006); para estas pruebas se excluyeron todas las especies que estuviesen representadas por 2 o menos individuos (11 en total), por lo que las pruebas se hicieron con base en 52 especies.

\section{Resultados}

Se capturaron un total de 2,367 especímenes pertenecientes a 63 especies, incluidas en 7 subfamilias (Apaturinae, Biblidinae, Charaxinae, Danainae, Limenitidinae, Nymphalinae y Satyrinae). Con base en lo obtenido con la ecuación de Clench para 

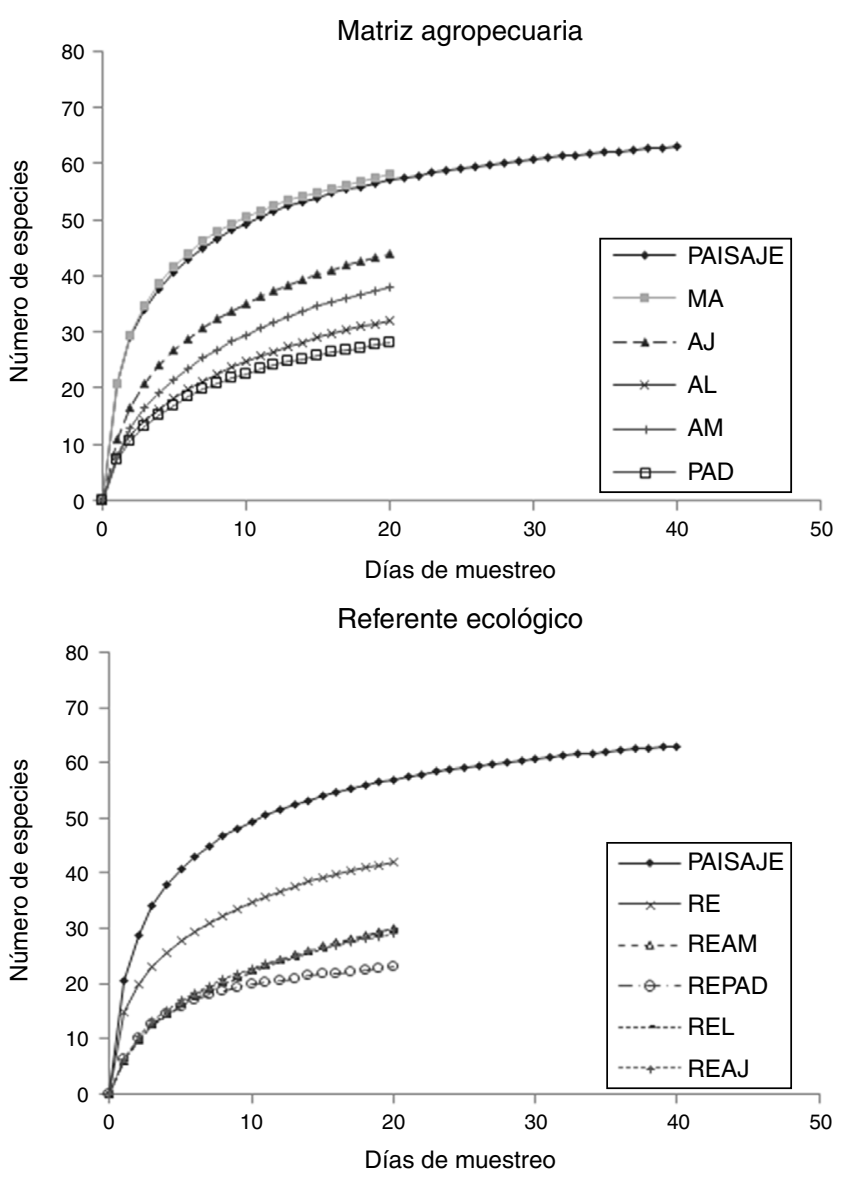

Figura 1. Curvas de rarefacción de especies para las Nymphalidae de la sierra de Tenosique, Tabasco. a) Unidades de paisaje del referente ecológico (RE), bosque referente para vegetación secundaria madura (REAM), bosque referente para vegetación secundaria joven (REAJ), bosque referente para potreros con árboles dispersos (REPAD), bosque alterado linealmente por rutas de acceso o senderos (REL). b) Matriz agropecuaria (MA): potreros con árboles dispersos $(\mathrm{PAD})$, árboles en línea $(\mathrm{AL})$, vegetación secundaria joven $(\mathrm{AJ})$, vegetación secundaria madura (AM).

cada curva de rarefacción, el muestreo de todo el paisaje registró el $94 \%$ del total de especies esperadas (67 especies). A nivel RE se registraron $42(91 \%)$ especies de las 46 esperadas, en MA se capturaron 58 (91\%) de 64 esperadas; así, el esfuerzo de muestreo se puede considerar adecuado al obtenerse un porcentaje mayor al 80\% de lo esperado (González-Valdivia et al., 2012). En ningún caso, las UP alcanzaron la riqueza de la MA o la del RE. Mientras que en MA la riqueza siguió la tendencia de la curva del paisaje (fig. 1a), en RE la riqueza estuvo por debajo de esta curva (fig. 1b).

La riqueza y la abundancia de mariposas difirieron entre UP $(\mathrm{F}=2.44, \mathrm{gl}=7, p=0.049 ; \mathrm{F}=8.83, \mathrm{gl}=7, p<0.001$, respectivamente), al igual que entre $\mathrm{RE}$ y $\mathrm{MA}(\mathrm{F}=5.96, \mathrm{gl}=1, p=0.021$; $\mathrm{F}=28.90, \mathrm{gl}=1, p<0.001$, respectivamente). Los extremos para la riqueza se encontraron en REPAD con 24 especies y AJ con 45 especies. La diversidad fue estadísticamente similar entre unidades $(\mathrm{F}=1.90, \mathrm{gl}=7, p=0.12)$, con valores desde medios (1.89) hasta altos (3.03) para el índice Shannon-Wiener. Solo se encontró dominancia de especies de mariposas en los PAD y los AL (índice Simpson $D=0.29$ ). La equitatividad en estos 2
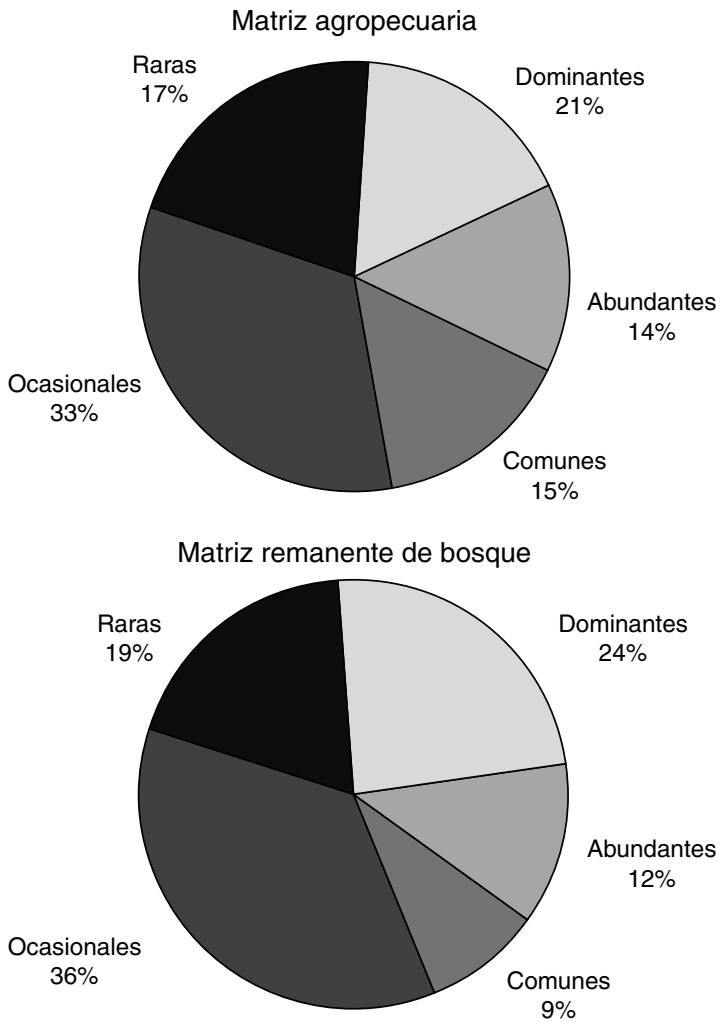

Figura 2. Proporción de las categorías de abundancia asignada a las especies de Nymphalidae en la matriz agropecuaria y el referente ecológico en la sierra de Tenosique, Tabasco, México.

últimos ambientes del paisaje fue baja (índice de Pielou $=0.57$ y 0.55 , respectivamente) con el género Hamadryas (Biblidinae) como el más abundante. Las demás comunidades de MA y las de RE se distribuyeron equitativamente (tabla 2).

Las proporciones de las abundancias por categoría fueron similares entre los 2 ecomosaicos. En la MA y en el RE se presentaron especies raras y ocasionales en un 50 y $55 \%$ respectivamente. La mayor diferencia entre estos 2 ecomosaicos se encontró en la categoría de especies comunes, siendo el $15 \%$ para MA y solo el $9 \%$ para RE (fig. 2). La abundancia para cada especie por unidad del paisaje se detalla en el Apéndice.

Las variables estructurales y ambientales de las UP se correlacionaron significativamente $(p<0.05)$ con la cantidad de individuos capturados. La altura media del dosel $(r=-0.78$, $p<0.001)$, la cobertura del dosel $(\mathrm{r}=-0.65, p<0.001)$, la temperatura $(\mathrm{r}=0.64, p<0.001)$, la iluminación $(\mathrm{r}=0.61, p<0.001)$ y la altitud $(\mathrm{r}=-0.64, p<0.001)$ fueron significativas. La riqueza de especies no mostró correlación con las variables estructurales y ambientales $(\mathrm{r}<0.28)$, aunque fue numéricamente mayor en las unidades de vegetación secundaria (AJ y AM) respecto a las demás unidades del paisaje incluyendo las de RE, que en general, fueron tan ricas en especies como los PAD y AL.

\section{Diversidad beta y relaciones ecológicas}

$\mathrm{Al}$ analizar la similitud de especies de mariposas entre las UP mediante el Anosim, se encontraron diferencias significativas 
Tabla 2

Índices ecológicos para las comunidades de mariposas identificadas en las distintas unidades y ecomosaicos del paisaje en la Sierra de Tenosique, Tabasco. Entre paréntesis las medias y desviaciones estándar para la riqueza por repetición de la unidad.

\begin{tabular}{|c|c|c|c|c|c|c|c|c|}
\hline \multirow[t]{3}{*}{ Índice } & \multicolumn{4}{|c|}{ Matriz agropecuaria } & \multicolumn{4}{|c|}{ Referente ecológico } \\
\hline & \multicolumn{4}{|c|}{ Unidad de paisaje } & \multicolumn{4}{|c|}{ Unidad de paisaje } \\
\hline & PAD & $\mathrm{AL}$ & AJ & $\mathrm{AM}$ & REAM & REAJ & REPAD & REL \\
\hline Riqueza observada & $28 \mathrm{ab}(7 \pm 2)$ & $32 \mathrm{ab}(7 \pm 3)$ & $45 \mathrm{a}(10 \pm 3)$ & $38 \mathrm{ab}(8 \pm 2)$ & $30 a b(5 \pm 3)$ & $29 \mathrm{ab}(6 \pm 2)$ & $24 \mathrm{~b}(6 \pm 3)$ & $30 \mathrm{ab}(6 \pm 2)$ \\
\hline Riqueza esperada & 33 & 38 & 53 & 49 & 38 & 36 & 27 & 37 \\
\hline Abundancia & $459 \mathrm{a}$ & $522 \mathrm{a}$ & $446 \mathrm{a}$ & $226 b$ & $169 \mathrm{~b}$ & $187 \mathrm{~b}$ & $171 \mathrm{~b}$ & $187 \mathrm{~b}$ \\
\hline Shannon H' & 1.91 & 1.89 & 2.79 & 3.03 & 2.82 & 2.74 & 2.75 & 2.66 \\
\hline Simpson D & 0.29 & 0.29 & 0.12 & 0.07 & 0.08 & 0.09 & 0.08 & 0.10 \\
\hline Equitatividad & 0.57 & 0.55 & 0.73 & 0.83 & 0.83 & 0.81 & 0.87 & 0.78 \\
\hline
\end{tabular}

AJ: vegetación secundaria joven; AL: árboles en línea; AM: vegetación secundaria madura; PAD: potreros con árboles dispersos; REAJ: bosque referente para vegetación secundaria joven; REAM: bosque referente para vegetación secundaria maduras; REL: bosque alterado linealmente por rutas de acceso o senderos; REPAD: bosque referente para potreros con árboles dispersos.

$(\mathrm{r}=0.56, p=0.001)$. Mediante las UPGMA se separaron 3 grupos, el primero incluyó a todas las unidades de RE, el segundo a las unidades de vegetación secundaria (AJ y AM) y el tercero a los potreros, tanto con árboles dispersos (PAD) como en línea (AL). Las unidades de RE tuvieron más del $75 \%$ de similitud entre sí. Los AJ y AM compartieron más del 70\% de las especies y las unidades de PAD y AL fueron similares en más del 80\% (fig. 3). El UPGMA separó a RE de MA, ya que aunque 37 especies (el 59\% de las 63 totales) fueron compartidas, 21 fueron exclusivas para MA y solo 5 especies para RE (tabla 3, Apéndice).

De acuerdo al ACC, la subfamilia Danainae, en particular las especies Napeogenes tolosa y Oleria paula se asociaron a RE, mientras que especies de las subfamilias Charaxinae,
Morphinae y Satyrinae se encontraron preferentemente en hábitats con arbolado denso (RE), aunque también fueron frecuentes en la vegetación secundaria (AJ o AM), como es el caso de Archaeoprepona demophon centralis, A. demophoon gulina, Morpho helenor octavia y Pareuptychia metaleuca metalecuca. La mayoría de las especies de las subfamilias Biblidinae y Limenitidinae, por ejemplo Adelpha iphicleola, Biblis hyperia aganisa, Hamadryas amphinome mexicana, H. feronia farinulenta, $H$. februa ferentina y $H$. guatemalena marmarice, se encontraron en los hábitats alterados. De Morphinae, solo Opsiphanes cassina fabricii se localizó en hábitats abiertos. La mariposa Smyrna blomfildia datis (Nymphalinae) se distribuyó ampliamente en el paisaje, comportándose como generalista en términos de uso de los hábitats que proporcionan las distintas UP

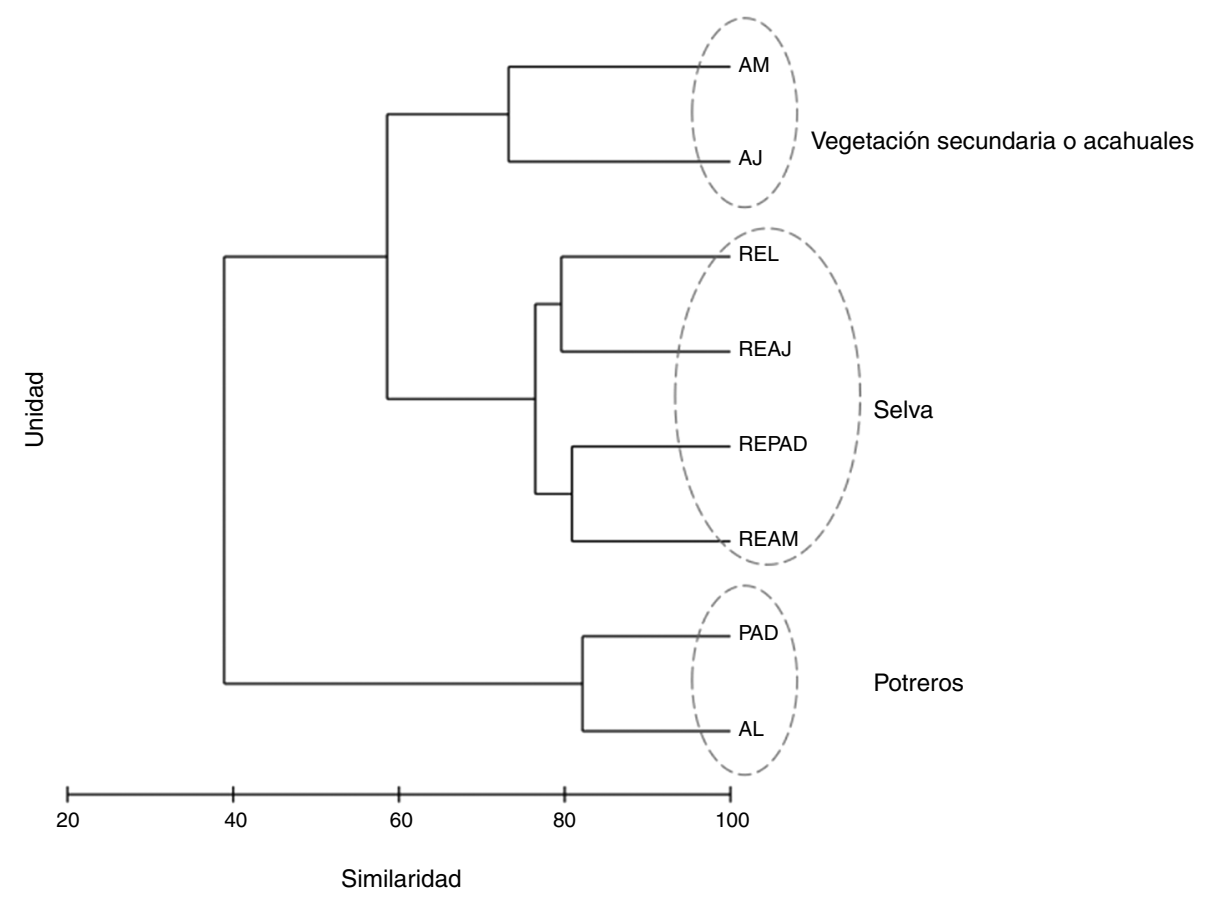

Figura 3. Análisis de agrupamiento utilizando el índice Bray-Curtis para las comunidades de mariposas frugívoras (Nymphalidae: Papilionoidea) de la sierra de Tenosique, Tabasco, México. AJ: vegetación secundaria joven; AL: árboles en línea; AM: vegetación secundaria madura; PAD: potreros con árboles dispersos; REAJ: bosque referente para vegetación secundaria joven; REAM: bosque referente para vegetación secundaria maduras; REL: bosque alterado linealmente por rutas de acceso o senderos; REPAD: bosque referente para potreros con árboles dispersos. 
Tabla 3

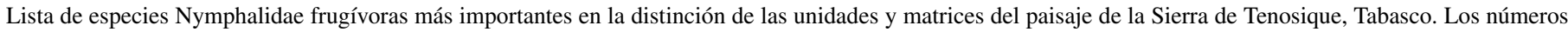

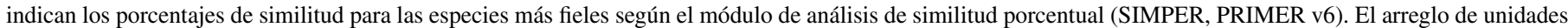
del paisaje va de menos arbolado a más arbolado (de izquierda a derecha).

\begin{tabular}{|c|c|c|c|c|c|c|c|c|}
\hline \multirow[b]{2}{*}{ Unidad de paisaje } & \multicolumn{3}{|c|}{ Matriz agropecuaria } & \multicolumn{5}{|c|}{ Referente ecológico } \\
\hline & PAD & AL & $\mathrm{AJ}$ & $\mathrm{AM}$ & REL & REAM & REAJ & REPAD \\
\hline Opsiphanes cassina fabricii & & 1.8 & & & & & & \\
\hline Hermeuptychia hermes & 3.3 & & & & & & & \\
\hline Hamadryas amphinome mexicana & 1.8 & & 1.7 & 8.1 & & 2.2 & & \\
\hline Hamadryas guatemalena marmarice & 15.1 & 6.1 & 5.0 & 5.1 & & & & \\
\hline Hamadryas feronia farinulenta & 48.2 & 59.4 & 44.6 & 4.9 & & & & \\
\hline Hamadryas februa ferentina & 19.2 & 21.9 & 13.2 & 26.6 & & & & \\
\hline Catonephele numilia esite & & & 1.9 & 2.3 & & & & \\
\hline Biblis hyperia aganisa & & 3.5 & 3.3 & & & & & \\
\hline Pteronymia cotytto cotytto & & & 3.8 & 13.4 & & & & \\
\hline Morpho helenor octavia & & & & 6.1 & 3.2 & 3.2 & & 5.4 \\
\hline Pareuptychia m. metaleuca & & & & 11.7 & 6.1 & 9.0 & 13.0 & 21.6 \\
\hline Archaeoprepona d. centralis & & & 1.6 & 2.5 & 4.7 & 18.2 & 16.7 & 7.6 \\
\hline Archaeoprepona d. gulina & & & 1.3 & 3.0 & & 3.9 & & 9.7 \\
\hline Tigridia acesta $\mathrm{ssp} . \mathrm{n}$ & & & & 3.3 & & & & 8.7 \\
\hline Catonephele mexicana & & & 3.7 & & 3.0 & 2.1 & & \\
\hline Taygetis virgilia & & & 1.91 & & 17.8 & 18.1 & 4.1 & 5.3 \\
\hline Oleria paula & & & & & 21.2 & 8.4 & 11.4 & 16.7 \\
\hline Napeogenes tolosa tolosa & & & & & 9.8 & 7.0 & 10.6 & 6.1 \\
\hline Caligo uranus & & & & & 5.4 & 3.2 & 7.9 & 2.7 \\
\hline
\end{tabular}

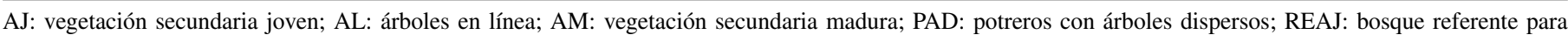

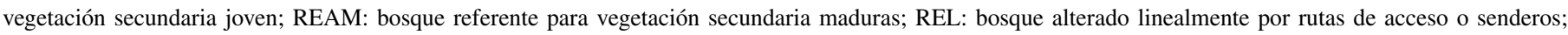
REPAD: bosque referente para potreros con árboles dispersos.

(fig. 4, Apéndice). Es importante indicar que todas las especies identificadas presentan amplia distribución geográfica y ninguna fue endémica o de distribución restringida.

El color corteza típico del género Hamadryas (Biblidinae) estuvo relacionado con la MA en general, pero en particular con potreros y AJ. De forma similar, especies como Adelpha basilioides, A. iphicleola, A. naxia (patrón Adelpha),
Historis a. acheronta, Historis odius dious, Nica flavilla bachiana, Siderone galanthis, Temenis laothoe hondurensis (naranja del dosel), Caligo telamonius memnon, Morpho helenor octavia (reflectante del sotobosque), Memphis forreri, $M$. mora orthesia, M. moruus boisduvali (reflectante del dosel), así como Biblis hyperia aganisa (patrón negro/rojo), se asociaron a ambientes de moderadamente alterados (AJ y AM) a

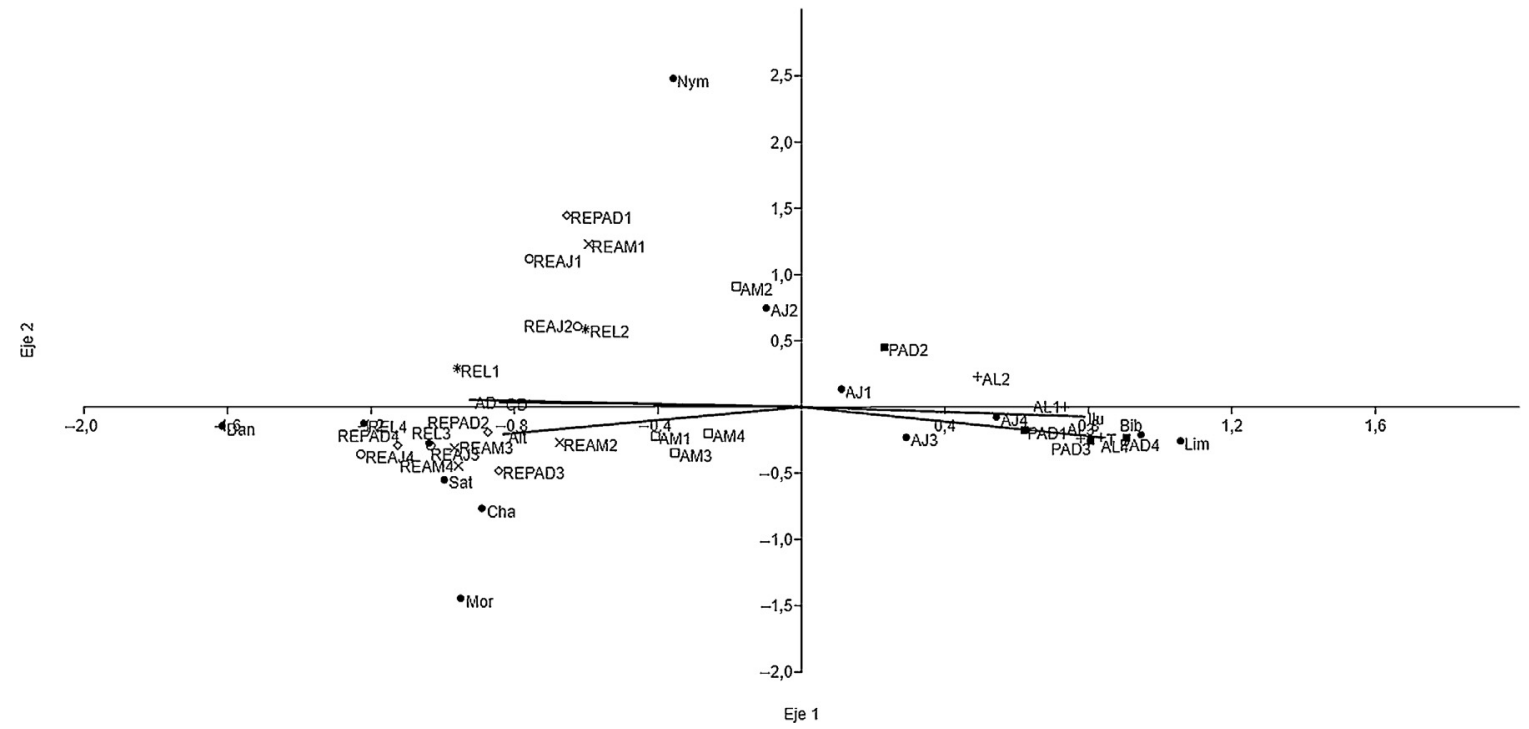

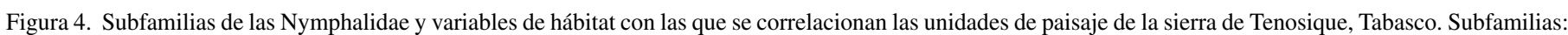

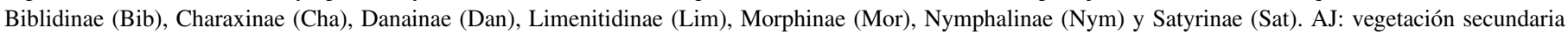

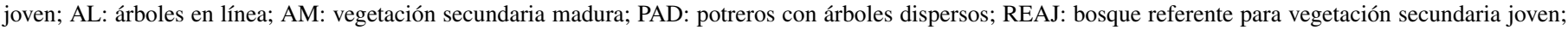

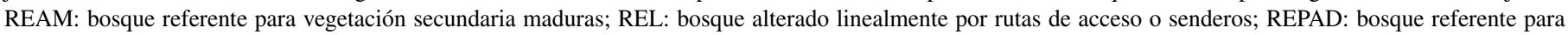
potreros con árboles dispersos. 


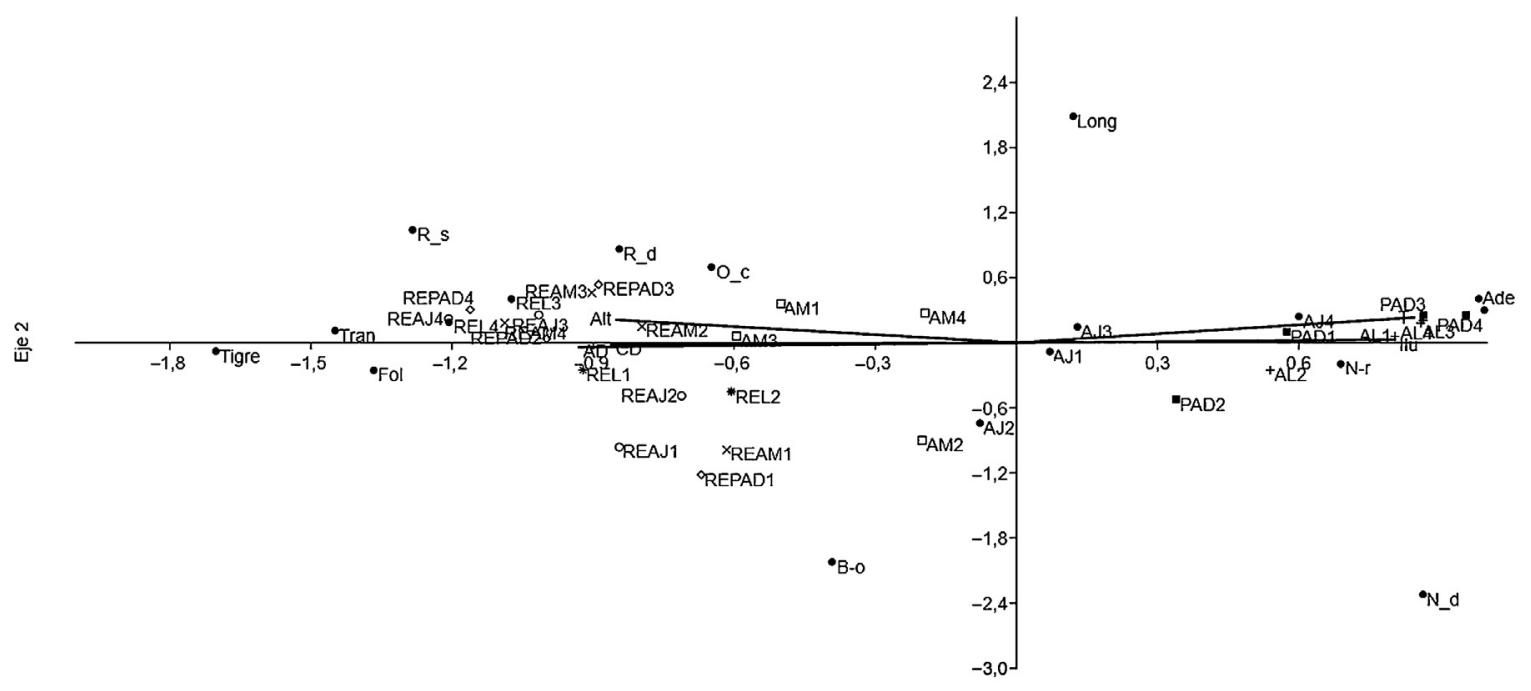

Eje 1

Figura 5. Patrones de coloración críptica de Nymphalidae y variables ambientales para cada unidad de paisaje de la Sierra de Tenosique, Tabasco, México. Categorías de color: adelpha (Ade), bandas longitudinales (Long), banda oblicua (B-o), corteza (Corteza), follaje (Fol), naranja del dosel (N-d), negro/rojo (N-r), oscuro críptico $(\mathrm{O}-\mathrm{c})$, reflectante del dosel (R-d), reflectante del sotobosque (R-s), tigre (Tigre) y transparente (Tran). Variables ambientales (arial): T: temperatura $\left({ }^{\circ} \mathrm{C}\right)$, Alt: altitud (m snm), Ilu: energía solar (MJ/m²/año), CD: cobertura de dosel (\%), AD: altura del dosel (m). AJ: vegetación secundaria joven; AL: árboles en línea; AM: vegetación secundaria madura; PAD: potreros con árboles dispersos; REAJ: bosque referente para vegetación secundaria joven; REAM: bosque referente para vegetación secundaria maduras; REL: bosque alterado linealmente por rutas de acceso o senderos; REPAD: bosque referente para potreros con árboles dispersos.

muy alterados (PAD y AL). Los patrones de coloración tigre y transparente, característicos de Danainae, abundaron en RE. Los Satyrinae de tamaño pequeño y color oscuro críptico fueron más comunes en hábitats ligeramente alterados (AM), aunque algunos de medianas dimensiones, como Manataria hercyna maculata o Taygetis mermeria excavata, se encontraron únicamente en RE. Los demás grupos ocuparon más ampliamente el paisaje destacándose el patrón de banda oblicua presentado por especies de las subfamilias Morphinae y Nymphalinae (fig. 5, Apéndice). Los ejes 1 y 2 del ACC explicaron más del 98.8\% de la relación subfamilia/ambiente y el $96 \%$ de la relación patrón de color/ambiente (para ambos casos $p=0.001$; figs. 4 y 5).

Las coloraciones que aportaron más a la diferenciación entre unidades conservadas y alteradas fueron los patrones tigre, transparente, reflectante sotobosque (indicadoras de áreas conservadas), corteza, naranja del dosel, negro/rojo, Adelpha (asociados al ambiente alterado). Las especies que incidieron más en la similitud entre UP y ecomosaicos (RE y MA) presentaron patrones de color banda oblicua, reflectante del dosel y oscuro críptico (Apéndice).

\section{Discusión}

En Tabasco se han registrado 115 especies de Nymphalidae frugívoras, con reportes para diferentes áreas protegidas del estado con valores de 69, 79, 66, 21 y 24 especies (MartínezGómez, 1994; Villegas-Segura, 1998; López-Quintero, 2002; Sánchez, 2002; Sosa-Vázquez, 2006, respectivamente). Con este estudio, la riqueza de Nymphalidae para el estado de Tabasco se incrementó en 5 nuevos registros: Archaeoprepona meander phoebus, Memphis aureola, Morpho helenor octavia, Morpho polyphemus luna y Tigridia acesta ssp. $\mathrm{n}$.
En el paisaje estudiado en Tenosique se encuentran representadas el $58 \%$ del total de mariposas de este gremio en Tabasco. Es de destacar que las especies identificadas en el paisaje del ejido han sido encontradas en áreas protegidas del estado de Tabasco (Martínez-Gómez, 1994; Villegas-Segura, 1998; Sánchez, 2002; López-Quintero, 2002; Sosa-Vázquez, 2006) o han sido mencionadas para Chiapas (De la Maza y De la Maza, 1993) y para Guatemala (Salinas-Gutiérrez, Méndez, Barrios, Pozo y Llorente-Bousquets, 2009). La riqueza de la zona es inferior a las 95 especies reportadas por Ragusso y Llorente-Bousquets (1990) para Los Tuxtlas, Veracruz, pero similar a las 63 especies reportadas en Calakmul, Campeche (Maya-Martínez, Pozo y May-Uc, 2005), la cual es considerada una de las mayores superficies forestales del trópico mexicano cuyos ecosistemas se caracterizan por su gran diversidad, riqueza y fragilidad (INECC, 2016). Todas las especies identificadas en el paisaje bajo estudio han sido reportadas en Guatemala y representan alrededor del 30\% de todas las Nymphalidae frugívoras registradas en ese país (Salinas-Gutiérrez et al., 2009).

Hubo variación en la composición de especies entre las UP. Sin embargo, el índice de diversidad fue similar entre estas -exceptuando los PAD y AL, ambos con baja diversidad- y los valores resultaron superiores a 2.24 expresados mediante el índice de Shannon-Wiener (diversidad alfa) que, de acuerdo con Ramírez-González (2006), pueden considerarse de medios a altos. Esto puede evidenciar que el manejo es adecuado para conservar al gremio de lepidópteros a escala del paisaje estudiado. No obstante, las diferencias entre la composición de especies de mariposas del RE respecto a la MA, también indican cambios provocados por el manejo del paisaje. En contraposición a Kerr, Perring y Currie (2006), y con base en la diversidad de mariposas del paisaje, los resultados apoyan la validez del modelo de disturbio intermedio (Connell, 1978). Así, el 
arreglo espacial y temporal de UP ejemplifica un sistema sometido a régimen de alteración intermedio capaz de mantener una diversidad de media a alta. La alteración y su régimen intermedio están representados por la cronosecuencia de vegetación secundaria, que en el territorio permite la recuperación del arbolado después de haber sido completa o parcialmente cortado para establecimiento del sistema de milpa. El periodo de descanso de la milpa supera en este caso los 25 años. En los extremos están los bosques con baja alteración y los potreros donde el periodo sin arbolado se prolonga, en general, a más de 10 años. No obstante, como afirman González-Espinosa, ReyBenayas, Ramírez-Marcial, Huston y Golicher (2004), también hay evidencia del impacto de la actividad antropogénica en las comunidades, al cambiar la composición de las mismas de una unidad de paisaje a otra.

La equitatividad registrada en las UP arboladas indica una tendencia al equilibrio entre las poblaciones de especies en estos elementos del paisaje, por lo que se puede asumir, como sugieren Wang, Chen y Chen (2009), que tienen capacidades de competencia similares. Sin embargo, esto a la larga podría provocar que algunas de ellas desaparezcan, particularmente si se incrementa la presión antrópica.

De manera similar a lo registrado para escarabajos estercoleros por Favila y Halffter (1997), en los pastizales inducidos se incrementaron la riqueza y la abundancia de las especies generalistas asociadas a hábitats abiertos (restringidas dentro del bosque a los claros, por ejemplo Hamadryas spp.), tal y como sugieren Sparrow et al. (1994), mientras que las especies asociadas a hábitats cerrados, del interior del bosque, disminuyen sus abundancias. Por su parte, la vegetación secundaria arbórea permite que algunas especies del bosque encuentren refugio en la matriz.

La mayor parte de las especies son consideradas raras debido a que sus abundancias son bajas (Ezcurra, 1990). Al respecto se sabe que la detección de especies raras o con presencia irregular se incrementa en función directa con la escala de análisis (Ezcurra, 1990; McGill et al., 2007). En el presente estudio, al analizar los datos considerando la escala de ecomosaico, se corroboró el efecto de la escala, ya que se registraron por arriba del $91 \%$ de las especies esperadas, que incluyen tanto especies raras como dominantes.

Los mayores registros de especies se obtuvieron en unidades de bosques secundarios jóvenes $(\mathrm{AJ})$, probablemente debido a una complejidad estructural intermedia generada a través de la sucesión ecológica, que permite que las especies de ambos extremos ambientales del paisaje puedan presentarse en estos ambientes. Es decir, existe una analogía con la teoría de disturbio intermedio propuesta por Connell (1978) o con el efecto del tipo dominio medio descrito por Colwell, Rahbek y Gotelli (2004). El traslape sucesional de las unidades de vegetación secundaria jóvenes y maduras, ubicadas entre las unidades de potreros con árboles y las unidades de bosque, les puede permitir funcionar como ecotono o «buffer», actuando como interfaces entre los hábitats conservados (RE) y aquellos más alterados (potreros). El tiempo de descanso prolongado favorece la recuperación del arbolado, diversificando en la vegetación secundaria tanto las fuentes de alimento como de refugio, sumado al efecto de los bosques adyacentes, que dispersan especies propias de estos hacia los barbechos.

El ACC ayudó a ilustrar la relación entre especies que se encuentran hacia condiciones ambientales con mayor temperatura e iluminación, con vegetación más abierta (unidades de potreros), así como otros grupos que se asocian a condiciones de arbolado más alto, denso y por tanto menos iluminado y más fresco hacia el interior de la selva, con una zona intermedia conformada por la vegetación secundaria, en las que se presentan diferencialmente ejemplares de ambas comunidades de mariposas en los ambientes que constituyen las condiciones extremas del gradiente de alteración.

Es importante destacar la variación en la composición de las comunidades relacionada con diferencias físico-estructurales entre las unidades. Esta influencia del ambiente en la composición de las comunidades de mariposas ha sido mencionado por Kusch, Goedert y Meyer (2005) para especies nocturnas de bosques mixtos europeos, influidas por diferencias en las condiciones del interior respecto a los bordes del bosque. En el paisaje estudiado pudo observarse una distinción entre las comunidades de Nymphalidae del bosque, de vegetación secundaria y de potreros.

La falta de correlación entre la riqueza de especies y las características físicas o las estructurales, así como la alta correlación de la abundancia de individuos con estas variables en las UP, confirman que la riqueza de especies no es un buen parámetro para determinar el impacto de las alteraciones del ecosistema sobre una comunidad. En consecuencia, es más relevante considerar la composición de especies y su abundancia relativa, tal como mencionan Aguilar (2005) y Uehara-Prado, Spalding y Lucci (2007).

La configuración del paisaje y lo heterogéneo de la MA, con hileras de árboles en los linderos y árboles dispersos, que funcionan, respectivamente, como corredores lineares o como escalones, facilitan el movimiento de mariposas entre fragmentos de bosques secundarios o primarios a través de los potreros, tal como sugiere Hadad (2000). De esta manera, especies que han sido mencionadas por De la Maza y Gutiérrez (1992), Pozo (2006) y Pozo, Salas-Suárez, Prado-Cuéllar y May-Uc (2009) como indicadoras de hábitat de bosques estables y conservadas, entre ellas a Archaeoprepona demophon centralis, A. demophoon gulina y Morpho helenor octavia, en este estudio aparecen indistintamente distribuidas en el bosque y en los bosques secundarios de la MA en el ejido, comprobando la permeabilidad al flujo de este grupo biológico, particularmente por el efecto de la vegetación secundaria. Esto tiene un significado relevante si se considera que la milpa, como sistema agroforestal rotacional, estaría propiciando en su prolongada fase de descanso o barbecho de la tierra el establecimiento de hábitats que pueden ayudar a la conservación de especies del bosque. Otras especies se confirman como características de áreas alteradas o con poca densidad en el arbolado, como sucede con Hamadryas februa ferentina, Hermeneuptychia hermes o Siproeta stelenes biplagiata. Solamente una especie, Smyrna blomfildia datis, pudo considerarse como de amplia y abundante presencia en todas las unidades y ecomosaicos del paisaje. 
En concordancia con Pozo et al. (2009), se confirma la discrepancia sobre las especies Memphis moruus boisduvali y Biblis hyperia aganisa, encontradas en unidades de ambos ecomosaicos del paisaje pero con mayor frecuencia en las zonas alteradas (vegetación secundaria), debilitando la evidencia de que prefieren hábitats conservados (De la Maza y Gutiérrez, 1992; Pozo, 2006). En el mismo sentido, Memphis forreri e Historis acheronta, mencionadas como de hábito forestal (Apaza et al., 2006; Pozo, 1999), ocuparon preferentemente la MA, asociándose principalmente a la vegetación secundaria. En Historis acheronta, puede considerarse que el hábito de alimentación preferencial de los estados inmaduros (Cecropia spp.) influye en donde se presentan los adultos (González-Valdivia et al., 2010), asumiendo que este género de árboles pioneros es indicador de sucesión temprana del bosque.

La riqueza registrada sugiere que la heterogeneidad de la MA es la característica que permite sostener hasta un 53\% de los Nymphalidae frugívoros asociados a hábitats conservados o poco alterados y hasta un $34 \%$ de especies que habitan preferentemente hábitats abiertos, además de las especies generalistas. Esto puede ocurrir debido a que la MA en el paisaje presenta franjas adyacentes de AJ y AM, donde algunas especies del bosque pueden establecerse. Esto facilita espacios donde prosperan las especies asociadas a hábitats alterados o abiertos, que dentro del bosque encuentran pocos sitios para su establecimiento (claros).

La riqueza de especies en el bosque y la MA aquí estudiados permiten considerar, de acuerdo con Schulze et al. (2004), que las mariposas pueden persistir en paisajes que combinan grandes fragmentos continuos de bosque maduro, junto a otros con sistemas de manejo que incluyan arbolado, como la vegetación secundaria (barbecho) y sistemas agroforestales. La conectividad al interior de este tipo de paisajes, con arbolado en arreglos lineales o dispersos entre áreas cubiertas con denso arbolado de sucesión secundaria, adyacentes a relictos de bosque primario, favorece el flujo de organismos e influye positivamente en las poblaciones.

Los resultados concuerdan con los de DeVries y Walla (2001), y las subfamilias que estos autores consideran relacionadas con áreas de bosque también se hallaron en el presente estudio. Por otro lado, Lencinas, Martínez, Anderson y Busso (2008) encontraron que muchas mariposas son generalistas de bosque, sea este conservado o secundario, lo que explicaría la similitud entre las unidades de RE y de $\mathrm{AM}$ en el paisaje aquí estudiado.

Se corroboró la propuesta de De la Maza y de la Maza (1993) y De la Maza y Soberón (1998) al demostrar la relación entre patrones de coloración de las mariposas diurnas y el grado de alteración del hábitat, confirmando que este análisis puede ser una herramienta útil para categorizar el impacto natural y/o antropogénico sobre los ecosistemas terrestres tropicales. No obstante, los resultados difieren en cuanto a la asignación de preferencias de hábitat de algunas especies, que De la Maza y de la Maza (1993) hicieron en su descripción de los grupos de color asociados a tipos de microhábitats en Chiapas, México, pero coinciden con los resultados de Apaza et al. (2006) en considerar a especies de Satyrinae, Danainae, Charaxinae y
Morphinae como indicadoras de áreas conservadas de bosque tropical, así como las especies de Biblidinae como indicadoras de alteración del ecosistema forestal primario. La jerarquía de subfamilia permitió distinguir los diferentes hábitats, funcionando como una herramienta con potencial de uso en la evaluación y monitoreo de impactos a los ecosistemas neotropicales terrestres. En este punto, se debe aclarar que hay excepciones y que la preferencia de hábitat por las especies de una subfamilia, en este caso de estudio, es una tendencia mayoritaria, mas no absoluta.

La utilidad de los niveles jerárquicos (especies y subfamilias) como indicadores ecológicos, aquí demostrada, coincide con los resultados de Cleary (2004) y Cleary, Suharsono y Hoeksema (2006). Esto es relevante dentro de la planificación de procesos de monitoreo o diagnóstico, haciendo recomendable a las Nymphalidae frugívoras como grupo funcional indicador. Algunas especies raras pueden haber sido subvaloradas como indicadoras ecológicas, especialmente Morpho polyphemus luna, que por observación de su comportamiento en campo, donde se presentó regularmente volando alto en el dosel arbóreo, presentó mayor valor de fidelidad por el bosque y los bosques secundarios maduros en el paisaje bajo estudio, pero su registro mediante las trampas fue muy baja. No obstante, continúa siendo una especie indicadora asociada a especies frugívoras que coexisten dentro de los mismos hábitats preferenciales.

La composición de Nymphalidae frugívoras respondió a las modificaciones del hábitat de selva original producto del manejo del ambiente que los pobladores hacen en el territorio. Esto pudo ser medido mediante los diferentes análisis multivariados realizados (análisis de cúmulos y ACC) y verificado con base tanto en las especies y subfamilias, como en los patrones de coloración de las especies asociadas a diferentes UP. El alto valor de los ejes 1 y 2 del ACC (98.8 y 96\%) confirma la relación patrón de color/ambiente. Esto significa que las mariposas muestran una tendencia a volar en ambiente particulares.

Hubo mayor diversidad de mariposas en la MA que en el RE e incluso 21 especies fueron registradas solamente en la MA. Las 5 especies encontradas exclusivamente en RE constituyen un grupo valioso para evaluar y monitorear el estado de conservación de los remanentes del bosque tropical lluvioso en la zona, mientras el género Hamadryas es útil para detectar efectos de mayor alteración de este ecosistema.

El manejo del paisaje estudiado puede aún considerarse, en su actual arreglo de ecomosaico con diferentes UP, como ecológicamente adecuado para la conservación de las especies de mariposas diurnas frugívoras. Esto probablemente es debido que el paisaje local contiene una masa de bosque continua y fragmentos dispersos, conectados por vegetación secundaria (alguna mayor de 25 años), que en su conjunto cubren aproximadamente el 50\% del territorio estudiado, así como por la presencia de árboles dispersos en los potreros y $\mathrm{AL}$ en los perímetros de los mismos, como parte del manejo que se hace en esta región. $\mathrm{Si}$ este modelo de uso de suelo se extendiese hacia las partes más alteradas (potreros sin arbolado que dominan el territorio del estado), se podría revertir el deterioro ecológico actual del estado de Tabasco, promoviendo la restauración diversificada de comunidades forestales y la incorporación de prácticas 
agroforestales, que fomente la complejidad del paisaje e incrementen la conectividad ecológica en el territorio.

\section{Agradecimientos}

A los campesinos de Niños Héroes de Chapultepec por permitir el acceso a sus parcelas. A Vicente López Moreno por su apoyo en campo. A Fomix-Conacyt, Tabasco, por su apoyo financiando el proyecto «Especies forestales de uso múltiple: caracterización de sus usos y potencial de aplicación en planes de fomento forestal, agroforestal y silvopastoril en el municipio de Tenosique, Tabasco, TAB-2007-C09-74820». A ECOSUR por su apoyo en infraestructura. Al personal del Museo de Zoología de la Unidad Chetumal de Ecosur, en especial a Noemí Salas por el apoyo en la identificación de las mariposas. A EAGE «Pbro. Francisco Luis Espinoza Pineda», la UNN, la UPONIC, al Gobierno de Nicaragua y a la DIA-SRE, México, por haber respaldado los estudios del primer autor.
Apéndice. Abundancia relativa de Nymphalidae frugívoras por unidades de paisaje y total por especie en el paisaje de la Sierra de Tenosique, Tabasco.

Clasificación de patrones de coloración de acuerdo a De la Maza y de la Maza (1993). AJ: vegetación secundaria joven; AL: árboles en línea; AM: vegetación secundaria madura; PAD: potreros con árboles dispersos; REAJ: bosque referente para vegetación secundaria joven; REAM: referente para vegetación secundaria madura; REL: bosque alterado linealmente por rutas de acceso 0 senderos; REPAD: bosque referente para potreros con árboles dispersos. Categorías de abundancia: dominantes (D), abundante (A), común (C), ocasional (O) y rara (R). Patrón de coloración: adelpha (Ade), bandas longitudinales (Long), banda oblicua (B-o), corteza (Corteza), follaje (Fol), naranja del dosel (N-d), neptis (Neptis) negro-rojo (N-r), oscuro críptico (O-c), reflectante del dosel (R-d), reflectante del sotobosque (R-s), tigre (Tigre) y transparente (Tran).

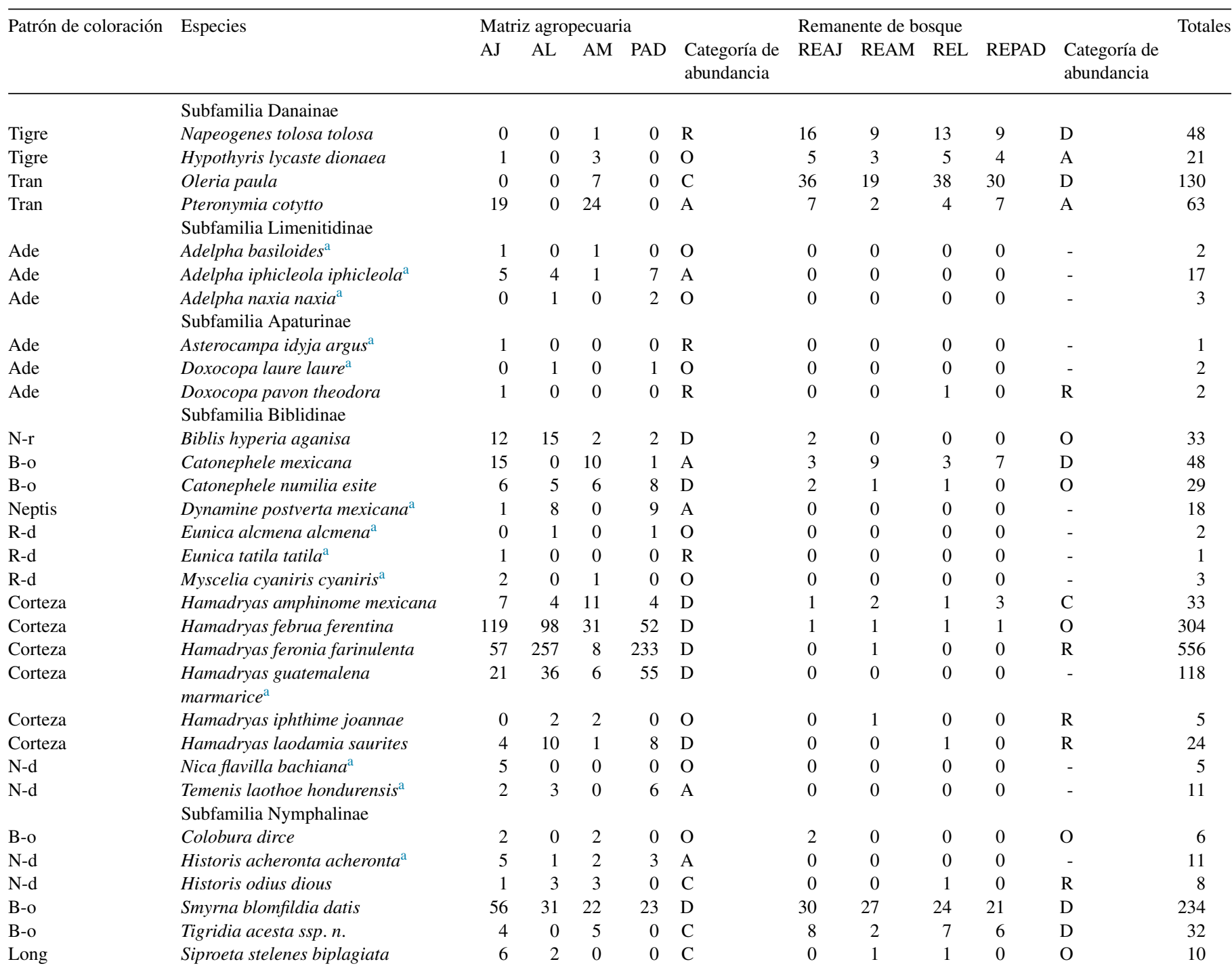


Apéndice (continuación)

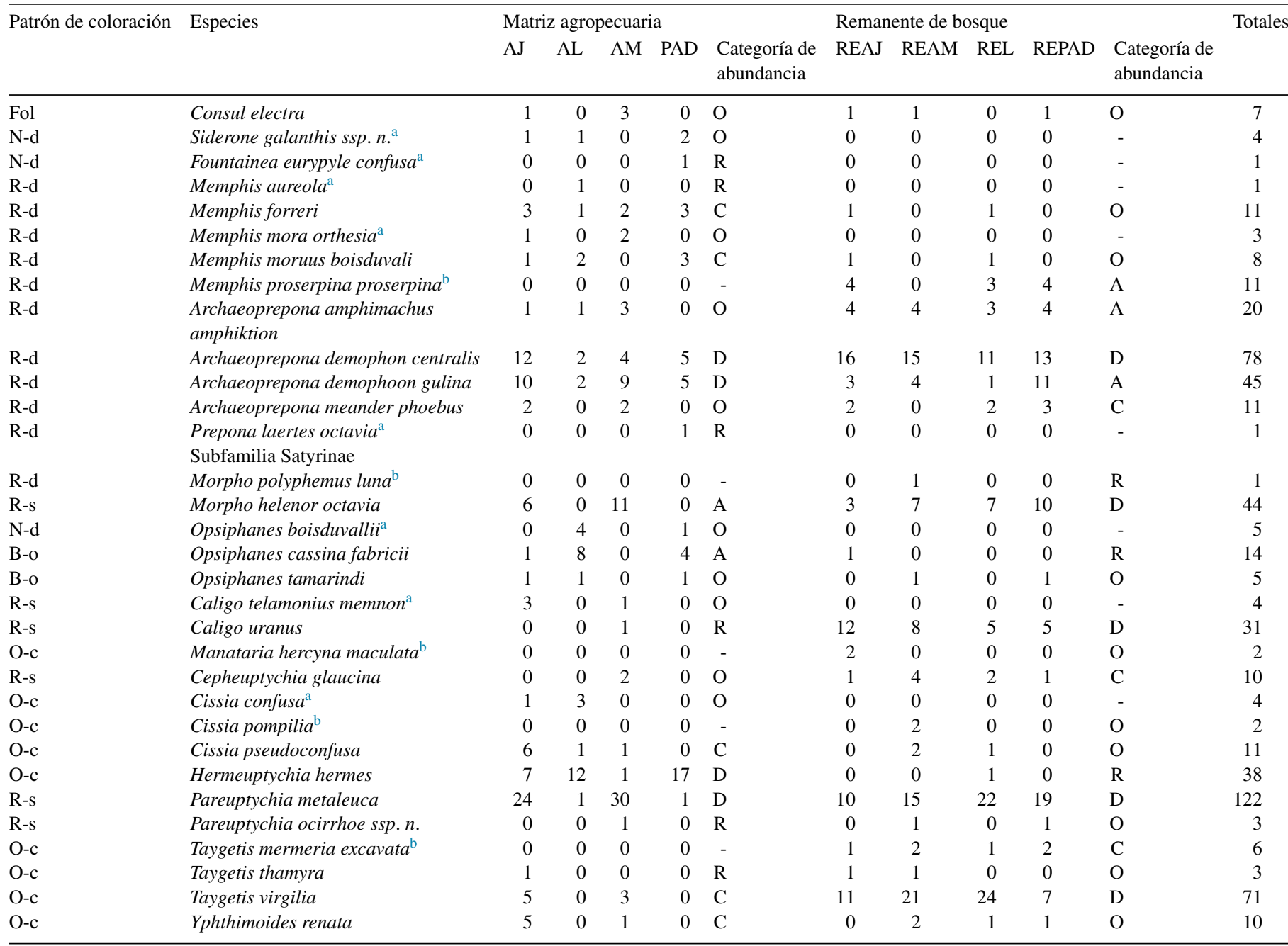

${ }^{\text {a }}$ Exclusivas de la matriz agropecuaria.

${ }^{\mathrm{b}}$ Exclusivas del referente de bosque.

\section{Referencias}

Aguilar, A. (2005). Los peces como indicadores de la calidad ecológica del agua. Revista Digital Universitaria, 6, 1-14.

Apaza, M. A., Osorio, F. y Pastrana, A. (2006). Evaluación del grado de amenaza al hábitat a través de bioindicadores (lepidópteros) en dos comunidades dentro del área de influencia del PN Anmi Madidi. Ciencia y Tecnología Agropecuaria, 1, 1-14.

Araujo, F. (2000). Nymphalid butterfly communities in an amazonian forest fragment. Journal of Research on the Lepidoptera, 35, 29-45.

Atauri, J. A. y de Lucio, J. V. (2001). The role of landscape structure in species richness distribution of birds, amphibians, reptiles and lepidopterans in Mediterranean landscapes. Landscape Ecology, 16, 147-159.

Beck, J., Kitching, I. y Linsenmair, E. (2006). Effects of habitat disturbance can be subtle yet significant: biodiversity of hawkmoth-assemblages (Lepidoptera: Sphingidae) in Southeast-Asia. Biodiversity and Conservation, 15, 465-486.

Bonebrake, T. C., Ponisio, L. C., Boggs, C. L. y Ehrlich, P. R. (2010). More than just indicators: a review of tropical butterfly ecology and conservation. Biological Conservation, 143, 1831-1841.

Box, G. y Cox, D. (1964). An analysis of transformations. Journal of Research of the Statistical Society, 26, 211-243.
Clarke, K. R. y Gorley, R. N. (2006). Primer v.6: user manual/tutorial. Plymouth routine in multivariate ecological research (Primer-e Ltd.). Plymouth, 182 Recuperado 18 Jun 2014, de: http://www.primer-e.com

Cleary, D. F. R. (2004). Assessing the use of butterflies as indicators of logging in Borneo at three taxonomic levels. Journal of Economic Entomology, 97 , 429-435.

Cleary, D. F. R., Suharsono y Hoeksema, B. W. (2006). Coral diversity across a disturbance gradient in the Pulau Seribu reef complex off Jakarta, Indonesia. Biodiversity and Conservation, 15, 3653-3674.

Clench, H. K. (1979). How to make regional lists of butterflies: some thoughts. Journal of the Lepidopterists' Society, 33, 216-231.

Colwell, R.K. (2013). EstimateS: statistical estimation of species richness and shared species from samples, ver. 8. Recuperado 15 Jun 2014, de: http://www.purl.oclc.org/estimates.

Colwell, R., Rahbek, C. y Gotelli, N. (2004). The mid-domain effect and species richness patterns: What have we learned so far? The American Naturalist, $163,1-23$.

Conabio (Comisión Nacional para el Conocimiento y Uso de la Biodiversidad). (2009). ¿Qué es la biodiversidad? México D.F., México: Comisión Nacional para el Conocimiento y Uso de la Biodiversidad. Recuperado 15 Ene 2016, de: http://www.biodiversidad.gob.mx/biodiversidad/que_es_.html.

Connell, J. H. (1978). Diversity in tropical rain forests and coral reefs. Science, $199,1302-1310$ 
Coomes, O. T., Grimard, F. y Burt, G. J. (2000). Tropical forests and shifting cultivation: Secondary forest fallow dynamics among traditional farmers of the Peruvian Amazon. Ecological Economics, 32, 109-124.

De la Maza, R. y de la Maza, J. (1993). Mariposas de Chiapas. Gobierno del Estado de Chiapas, Chiapas, México. Tuxtla Gutiérrez: Espejo de Obsidiana Ediciones.

De la Maza, R. y Gutiérrez, D. (1992). Ropaloceros de Quintana Roo: su distribución, origen y evolución. Revista de la Sociedad Mexicana de Lepidopterología, 15, 3-43.

De la Maza, R. y Soberón, J. (1998). Morphological grouping of Mexican butterflies in relation to habitat association. Biodiversity and Conservation, 7 , 927-944.

DeFries, R., Rudel, T., Uriarte, M. y Hansen, M. (2010). Deforestation driven by urban population growth and agricultural trade in the twenty-first century. Nature Geoscience, 3, 178-181.

Delta-T Devices, Ltd. (1999). HemiView manual revision number 2.1. Cambridge: Delta-T Devices, Ltd.

DeVries, P. y Walla, T. (2001). Species diversity and community structure in Neotropical fruit-feeding butterflies. Biological Journal of the Linnean Society, 74, 1-15.

Ezcurra, E. (1990). ¿Por qué hay tantas especies raras? La riqueza y rareza biológicas en las comunidades naturales. Ciencias, 4, 82-88.

Favila, M. y Halffter, G. (1997). The use of indicator groups for measuring biodiversity as related to community structure and function. Acta Zoológica Mexicana (nueva serie), 72, 1-25.

Ford, A. y Nigh, R. (2010). The milpa cycle and the making of the Maya forest garden. Research Reports in Belizean Archaeology, 7, 183-190.

Fox, J., Truong, D. M., Rambo, A. T., Tuyen, N. P., Cuc, L. T. y Leisz, S. (2000). Shifting cultivation: a new old paradigm for managing tropical forests. BioScience, 50, 521-528.

Freitas, A. V. L., Iserhard, C. A., Santos, J. P., Carreira, J. Y. O., Ribeiro, D. B., Melo, D. H. A., et al. (2014). Studies with butterfly bait traps: an overview. Revista Colombiana de Entomología, 40, 209-218.

Gómez-Pompa, A. y Kaus, A. (1999). From pre-Hispanic to future conservation alternatives: lessons from Mexico. Proceedings of the National Academy of Sciences USA, 96, 5982-5986.

González-Espinosa, M., Rey-Benayas, J. M., Ramírez-Marcial, M., Huston, M. A. y Golicher, D. (2004). Tree diversity in the northern Neotropics: regional patterns in highly diverse Chiapas. Mexico. Ecography, 27, $741-756$.

González-Valdivia, N. A., Arriaga-Weiss, S., Ochoa-Gaona, S., Ferguson, B. G., Kampichler, C. y Pozo, C. (2012). Ensambles de aves diurnas a través de un gradiente de perturbación en un paisaje en el sureste de México. Acta Zoológica Mexicana (nueva serie), 28, 237-269.

González-Valdivia, N. A., Ochoa-Gaona, S., Pozo, C. y Dénommée-Patriganni, L. (2010). Catálogo de mariposas Rhopalocera diurnas en el ejido Niños Héroes de Chapultepec, Tenosique, Tabasco, México, con potencial de explotación, cría y comercialización. Villahermosa: El Colegio de la Frontera Sur.

Hadad, N. (2000). Corridor length and patch colonization by a butterfly, Junonia coenia. Conservation Biology, 14, 738-745.

Hammer, Ø., Harper, D. y Ryan, P. (2001). PAST: Paleontological Statistics Software Package for education and data analysis. Paleontología Electrónica, 4 , $1-9$.

Hanski, I. (1998). Metapopulation dynamics. Nature, 396, 41-49.

Hanski, I. y Gilpin, M. (1996). Metapopulation biology: ecology, genetics and extinction. Londres: London Academic Press.

Hartshorn, G. S. (1995). Ecological basis for sustainable development in tropical forests. Annual Review of Ecology and Systematics, 26, 155-175.

Harvey, C. y Haber, W. (1999). Remnant trees and the conservation of biodiversity in Costa Rican pastures. Agroforestry Systems, 44, 37-68.

Harvey, C., Komar, O., Chazdon, R., Ferguson, B., Finegan, B., Griffith, D., et al. (2008). Integrating agricultural landscapes with biodiversity conservation in the Mesoamerican hotspot. Conservation Biology, 22, 8-15.

Heppner, J. B. (2002). Mexican Lepidoptera biodiversity. Insectamundi, 16, $170-190$.

Hernández, M. I. M., Barreto, P. S. C. S., Costa, V. H., Creão-Duarte, A. J. y Favila, M. E. (2014). Response of a dung beetle assemblage along a reforestation gradient in Restinga forest. Journal of Insect Conservation, $18,539-546$.

Herrando-Pérez, S. (2002). Manual de ecología matemática. Un enfoque práctico al análisis multivariado (PCA, CLUSTER y MDS) para detectar patrones en ecología. Tenerife: Torbellino.

Higgs, E. (2003). Nature for design: people, natural processes and ecological restoration. Cambridge: Massachusetts Institute of Technology, MIT Press.

Holling, C. S. (2001). Understanding the complexity of economic, ecological, and social systems. Ecosystems, 4, 390-405.

Horner-Devine, M., Daily, G., Ehrlich, P. y Boggs, C. (2003). Countryside biogeography of tropical butterflies. Conservation Biology, 17, 168-177.

INECC (Instituto Nacional de Ecología y Cambio Climatico). (2016). Reserva de la Biosfera Calakmul. Instituto Nacional de Ecología, y Comisión Nacional para el Conocimiento y Uso de la Biodiversidad. Recuperado 15 Ene 2016, de: http://www2.inecc.gob.mx/publicaciones/libros/2/calakmul.html.

Isaac-Márquez, R., de Jong, B., Eastmond, A., Ochoa-Gaona, S. y Hernández, S. (2005). Estrategias productivas campesinas: un análisis de los factores condicionantes del uso de suelo en el Oriente de Tabasco (21) México: Universidad y Ciencia.

Kerr, J. T., Perring, M. y Currie, D. J. (2006). The missing Madagascan middomain effect. Ecology Letters, 9, 149-159.

Kitching, R., Orr, A., Thalib, L., Mitchell, H., Hopkins, M. y Graham, A. (2000). Moth assemblages as indicator of environmental quality remnants of upland Australian rain forest. Journal of Applied Ecology, 37, 284-297.

Krenn, H. W., Zulka, K. P. y Gatschnegg, T. (2001). Proboscis morphology and food preferences in nymphalid butterflies (Lepidoptera: Nymphalidae). Journal of Zoology, 254, 17-26.

Kusch, J., Goedert, C. y Meyer, M. (2005). Effects of patch type and food specializations on fine spatial scale community patterns of nocturnal forest associated Lepidoptera. Journal of Research on the Lepidoptera, 36, 67-77.

Lamas, G. (Ed.). (2004). Atlas of Neotropical Lepidoptera. Checklist: Part 4A, Hesperoidea-Papilionoidea. Gainesville: Association for Tropical Lepidoptera \& Scientific Publishers.

Legendre, P. (2008). Studying beta diversity: ecological variation portioning by multiple regression and canonical analysis. Journal of Plant Ecology, 1, 3-8.

Lencinas, M. V., Martínez, G., Anderson, C. y Busso, C. (2008). The value of timber quality forest for insect conservation on Tierra del Fuego Island compared to associated non-timber quality stands. Journal of Insect Conservation, 12, 461-475.

López-Quintero, P. (2002). Estructura de la superfamilia Papilionoidea (Insecta: Lepidoptera) en cuatro tipos de vegetación en la Reserva de la Biósfera Pantanos de Centla, Tabasco Tesis. Villahermosa, Tabasco: Universidad Juárez Autónoma de Tabasco.

MacArthur, R. H. y Wilson, E. O. (1967). The theory of island biogeography, Princeton, NJ: Princeton University Press.

Martínez-Gómez, L. (1994). Inventario de la división Rhopalocera (Lepidoptera: Frenatae) del Parque Estatal de Agua Blanca, Tabasco Tesis. Villahermosa, Tabasco: Universidad Juárez Autónoma de Tabasco.

Maya-Martínez, A., Pozo, C. y May-Uc, E. (2005). Las mariposas (Rhopalocera, Pieridae y Nymphalidae) de la selva alta subperennifolia de la región de Calakmul, México, con nuevos registros. Acta Zoológica Mexicana, 44, 123-143.

McGill, B., Etienne, R., Gray, J., Alonso, D., Anderson, M., Kassa, H., et al. (2007). Species abundance distributions: moving beyond single prediction theories to integration within an ecological framework. Ecology Letters, 10, 995-1015.

McGinley, M. (2014). Species richness. Recuperado 30 Mar 2016, de http://www.eoearth.org/view/article/156216.

Miller, J. Y. (1994). Behavior in butterflies as a means of conservation: comparison of insular and continental fauna. Florida Entomologist, 77, 74-84.

New, T. R. (1998). Butterfly conservation. South Melbourne: Oxford University Press.

Ochoa-Gaona, S., Pérez-Hernández, I. I. y de Jong, B. (2008). Fenología reproductiva de las especies arbóreas del bosque tropical de Tenosique, Tabasco, México. Revista de Biología Tropical, 56, 657-673.

Pinheiro, C. E. G. y Ortiz, J. V. C. (1992). Communities of fruit-feeding butterflies along a vegetation gradient in central Brazil. Journal of Biogeography, $19,505-511$. 
Pozo, C. (1999). Monitoreo de mariposas para la región de Calakmul, México. Capítulo III. En A. Carr y A. C. de Stoll (Eds.), Monitoreo biológico en la Selva Maya (pp. 28-35). Washington: Man and the Biosphere Program 6 Wildlife Conservation Society.

Pozo, C. (2006). Los Rhopalocera de la región de Calakmul, Campeche: métodos de estudio, fenología y su uso y monitoreo como indicadores de disturbio. México D.F: Universidad Nacional Autónoma de México.

Pozo, C., Llorente-Busquets, J., Luis-Martínez, A., Vargas-Fernández, I. y Salas, N. (2005). Reflexiones acerca de los métodos de muestreo para mariposas en las comparaciones biogeográficas. En J. Llorente y J. Morrone (Eds.), Regionalización geográfica en Iberoamérica y tópicos afines: primeras jornadas biogeográficas de la Red Iberoamericana de Biogeografía y Entomología Sistemática (RIBES XII.I. -CYTED) (pp. 203-215). México D.F: Universidad Nacional Autónoma de México.

Pozo, C., Luis-Martínez, A., Llorente-Busquets, J., Salas-Suárez, N., MayaMartínez, A., Vargas-Fernández, I., et al. (2008). Seasonality and phenology of the butterflies (Lepidoptera: Papilionoidea and Hesperioidea) of Mexico's Calakmul region. Florida. Entomologist, 91, 407-422.

Pozo, C., Salas-Suárez, N., Prado-Cuéllar, B. y May-Uc, E. (2009). Riqueza de mariposas diurnas (Lepidoptera: Rhopalocera) en el Santuario del Manatí y una propuesta para su uso en el monitoreo de ambientes terrestres del área. En J. Espinoza-Avalos, G. A. Islebe, y H. A. Hernández-Arana (Eds.), El sistema ecológico de la bahía de Chetumal/Corozal: costa occidental del Mar Caribe. Chetumal: El Colegio de la Frontera Sur.

Ragusso, R. A. y Llorente-Bousquets, J. (1990). The butterflies (Lepidoptera) of the Tuxtlas Mts., Veracruz, México, revisited: species-richness and habitat disturbance. Journal of Research on the Lepidoptera, 29, 105-133.

Ramírez-González, A. (2006). Ecología: métodos de muestreo y análisis de poblaciones y comunidades. Bogotá: Pontificia Universidad Javeriana.

Ramos, Z. (2004). Estructura y composición de un paisaje boscoso fragmentado: herramienta para el diseño de estrategias de conservación de la biodiversidad Tesis de Maestría. Turrialba, Costa Rica: Centro Agronómico Tropical de Investigación y Enseñanza.

Ricketts, T., Daily, G., Ehrlich, P. y Fay, J. (2001). Countryside biogeography of moths in a fragmented landscape: biodiversity in native and agricultural habitats. Conservation Biology, 15, 378-388.

Rossignol, M., Rossignol, L., Oldeman, R. y Benzine-Tizroutine, S. (1998). Struggle of life or the natural history of stress and adaptation. Treebook 1. Wageningen, Holanda: Treemail Publishers.

Salinas-Gutiérrez, J. L., Méndez, C., Barrios, M., Pozo, C. y Llorente-Bousquets, J. (2009). Hacia una síntesis de los Papilionoidea (Insecta: Lepidoptera) de Guatemala con una reseña histórica. Caldasia, 31, 407-440.

Sánchez, S. (2002). Lista preliminar de mariposas diurnas (Lepidoptera: Rhopalocera) del Parque Ecológico de La Chontalpa, Tabasco, México. Entomotropica, 17, 111-113.

Santos, J. P., Iserhard, C. A., Teixeira, M. O. y Romanowski, H. P. (2011). Fruit-feeding butterflies guide of subtropical Atlantic forest and Araucaria moist forest in the State of Rio Grande do Sul, Brazil. Biota Neotropica, 11, 253-274.

Schulze, C., Waltert, M., Kessler, P., Pitopang, R., Shahabuddin, G., Veddeler, D., et al. (2004). Biodiversity indicator groups of tropical land-use systems: comparing plants, birds, and insects. Ecological Applications, 15, 1321-1333.

Sedespa (Secretaría de Desarrollo Social y Protección del Ambiente). 1997. Ley de Protección Ambiental del Estado de Tabasco, Municipio de Tenosique. Gobierno del Estado de Tabasco. Villahermosa: Sedespa.
Semarnat (Secretaría de Medio Ambiente y Recursos Naturales). (2008). Decreto por el que se declara Área Natural Protegida con la categoría de área de protección de flora y fauna, la región conocida como Cañón del Usumacinta, localizada en el Municipio de Tenosique, en el Estado de Tabasco. Diario Oficial de la Federación, 22 de Septiembre de 2008, Gobierno del estado de Tabasco, Tabasco, México [última consulta 10 Ago 2013]. Disponible en: http://vlex.com.mx/vid/categoria-flora-faunausumacinta-tenosique-42835362.

Simberloff, D. (1974). Equilibrium theory of island biogeography and ecology. Annual Review of Ecology and Systematics, 5, 161-182.

Sosa-Vázquez, R. (2006). Composición de la superfamilia Papilionoidea (Insecta-Lepidoptera), en el centro ecoturístico de Yu Balcah, Tacotalpa, Tabasco, México Tesis. Villahermosa, Tabasco: Universidad Juárez Autónoma de Tabasco.

Sparrow, H., Sisk, T., Ehrlich, P. y Murphy, D. (1994). Techniques and guidelines for monitoring Neotropical butterflies. Conservation Biology, 8, 800-809.

Spellerberg, I. F. (2005). Monitoring ecological change. Cambridge: Cambridge University Press.

Stiling, P. D. (1999). Ecology: theories and applications (Third edition). Nueva Jersey: Prentice Hall.

Tobar, D., Ibrahim, M. y Casasola, F. (2007). Diversidad de mariposas en un paisaje agropecuario del Pacífico Central de Costa Rica. Agroforestería en las Américas, 45, 58-65.

Uehara-Prado, M., Spalding, K. y Lucci, A. (2007). Species richness, composition and abundance of fruit-feeding butterflies in the Brazilian Atlantic forest: comparison between a fragmented and a continuous landscapes. Global Ecology and Biogeography, 46, 43-54.

Villegas-Segura, I. (1998). Inventario de los Papilionoidea (Insecta: Lepidoptera) del Monumento Natural Cerro del Cocona, Teapa, Tabasco y algunos aspectos de su fenología Tesis. Villahermosa, Tabasco: Universidad Juárez Autónoma de Tabasco.

Wahlberg, N., Leneveu, J., Kodandaramaiah, U., Peña, C., Nylin, S., Freitas, A. V. L., et al. (2009). Nymphalid butterflies diversify following near demise at Cretaceous/Tertiary boundary. Proceeding of The Royal Society B (Biological Sciences), 276, 4295-4302.

Waltz, A. A. E., Covington, W. y Wallace, W. (2001). Butterfly response and successional change following ecosystem restoration. En R. K., Vance, C.B. Edminster, W. Covington, W. Wallace y J. A. Blake (Comps.), Ponderosa pine ecosystems restoration and conservation: steps toward stewardship. USDA Forest Service Proceedings 22, Rocky Mountain Research Station, Flagstaff, AZ.

Wang, Z., Chen, Y. F. y Chen, H. (2009). Functional grouping and establishment of distribution patterns of invasive plants in China using self-organizing maps and indicator species analysis. Archives of Biological Sciences, 61, 71-78.

Weibull, A. C., Bengtsson, J. y Nohlgren, E. (2000). Diversity of butterflies in the agricultural landscape: the role of farming system and landscape heterogeneity. Ecography, 23, 743-750.

Willmott, K. R. (2003). The genus Adelpha: its systematics, biology and biogeography (Lepidoptera: Nymphalidae: Limenitidini). Gainesville: Scientific Publishers.

Zonneveld, I. (1989). The land unit: a fundamental concept in landscape ecology, and its application. Landscape Ecology, 3, 67-83.

Zuur, A., Ieno, E. y Smith, G. (2007). Analyzing ecological data. En M. Gail, K. Krickeberg, J. Samet, A. Tsiatis, y W. Wong (Eds.), Statistics for biology and health (pp. 163-179). New York: Springer. 